In Volume for John Thompson's 70th birthday, in Progress in Galois Theory,

H. Voelklein and T. Shaska editors 2005 Springer Science, 51-85

\title{
Relating two genus 0 problems of John Thompson
}

\author{
Michael D. Fried
}

\begin{abstract}
Excluding a precise list of groups like alternating, symmetric, cyclic and dihedral, from 1st year algebra (\$7.2.3), we expect there are only finitely many monodromy groups of primitive genus 0 covers. Denote this nearly proven genus 0 problem as Problem ${ }_{2}^{g=0}$. We call the exceptional groups 0 -sporadic. Example: Finitely many Chevalley groups are 0 -sporadic. A proven result: Among polynomial 0-sporadic groups, precisely three produce covers falling in nontrivial reduced families. Each (miraculously) defines one natural genus $0 \mathbb{Q}$ cover of the $j$-line. The latest Nielsen class techniques apply to these dessins d'enfant to see their subtle arithmetic and interesting cusps.

John Thompson earlier considered another genus 0 problem: To find $\theta$ functions uniformizing certain genus 0 (near) modular curves. We call this Problem ${ }_{1}^{g=0}$. We pose uniformization problems for $j$-line covers in two cases. First: From the three 0-sporadic examples of Problem ${ }_{2}^{g=0}$. Second: From finite collections of genus 0 curves with aspects of Problem $\mathrm{m}_{1}^{g=0}$.
\end{abstract}

\section{CONTENTS}

1. Genus 0 themes

1.1. Production of significant genus 0 curves

1.2. Detailed results

2. Examples from Problem ${ }_{2}^{g=0}$

2.1. Review of Davenport's problem

2.2. Review of Nielsen classes

2.3. Davenport Pair monodromy groups

2.4. Müller's list of primitive polynomial monodromy groups

3. Explanation of the components for $\mathcal{H}_{7}^{\mathrm{DP}}, \mathcal{H}_{13}^{\mathrm{DP}}, \mathcal{H}_{15}^{\mathrm{DP}}$

3.1. Explicit difference sets

3.2. Difference sets give properties (3.1a) and (3.1b)

3.3. Completing property $(3.1 \mathrm{~b})$

3.4. The analog for $n=13$ and 15

4. $j$-line covers for polynomial $\mathrm{PGL}_{3}(\mathbb{Z} / 2)$ monodromy

4.1. Branch cycle presentation and definition field

4.2. b-fine moduli property

5. $j$-line covers for polynomial $\mathrm{PGL}_{3}(\mathbb{Z} / 3)$ monodromy

5.1. Degree 13 Davenport branch cycles $\left(g_{1}, g_{2}, g_{3}, g_{\infty}\right)$

1991 Mathematics Subject Classification. Primary 11F32, 11G18, 11R58, 14G32; Secondary 20B05, 20C25, 20D25, 20E18, 20F34.

Thanks to NSF Grant \#DMS-0202259, support for the Thompson Semester at U. of Florida. 
5.2. Proof of Prop. 5.1

6. Projective systems of Nielsen classes

6.1. Projective sequences of Nielsen classes

6.2. $\mathcal{N}_{2} \stackrel{\text { def }}{=}\left\{\mathrm{Ni}\left(G, \mathbf{C}_{2^{4}}\right)^{\text {in }}\right\}_{G \in \mathrm{Q}^{F_{2}}(2) \times{ }^{s} H_{2}}$

6.3. $\mathcal{N}_{3} \stackrel{\text { def }}{=}\left\{\mathrm{Ni}\left(G, \mathbf{C}_{ \pm 3^{2}}\right)^{\mathrm{in}}\right\}_{G \in \mathrm{Q}^{F_{2}}(3) \times{ }^{s} H_{3}}$

6.4. Projective cusp types

7. Relating Problem 1 and Problem ${ }_{2}^{g=0}$

7.1. Explicit equations

7.2. A piece of John Thompson's influence

References

\section{Genus 0 themes}

We denote projective 1-space $\mathbb{P}^{1}$ with a specific uniformizing variable $z$ by $\mathbb{P}_{z}^{1}$. This decoration helps track distinct domain and range copies of $\mathbb{P}^{1}$. We use classical groups: $D_{n}$ (dihedral), $A_{n}$ (alternating) and $S_{n}$ (symmetric) groups of degree $n$; $\mathrm{PGL}_{2}(K)$, Möbius transformations over $K$; and generalization of these to $\mathrm{PGL}_{u+1}(K)$ acting on $k$-planes, $0 \leq k \leq u-1$, of $\mathbb{P}^{u}(K)$ ( $K$ points of projective $u$-space). $\S 4.2$ denotes the space of four distinct unordered points of $\mathbb{P}_{z}^{1}$ by $U_{4}=$ $\left(\left(\mathbb{P}_{z}^{1}\right)^{4} \backslash \Delta_{4}\right) / S_{4}$. For $K$ a field, $G_{K}$ is its absolute Galois group (we infrequently allude to this for some applications).

A $g \in S_{n}$ has an index ind $(g)=n-u$ where $u$ is the number of disjoint cycles in $g$. Example: $(123)(4567) \in S_{7}$ has index $7-2=5$. Suppose $\varphi: X \rightarrow \mathbb{P}_{z}^{1}$ is a degree $n$ cover (of compact Riemann surfaces). We assume the reader knows about the genus $g_{X}$ of $X$ given a branch cycle description $\boldsymbol{g}=\left(g_{1}, \ldots, g_{r}\right)$ for $\varphi(\S 2.2 .1)$ : $2\left(n+g_{X}-1\right)=\sum_{i=1}^{r} \operatorname{ind}\left(g_{i}\right)([\mathbf{V} \ddot{\mathbf{9} 96}, \S 2.2]$ or [Fr05, Chap. 4] $)$.

1.1. Production of significant genus 0 curves. Compact Riemann surfaces arose to codify two variable algebraic relations. The moduli of covers is a refinement. For a given genus, this refinement has many subfamilies, with associated discrete invariants. Typically, these invariants are some type of Nielsen class $(\S 2.2)$. The parameter space for such a family can have any dimension. Yet, we benefit by comparing genus $g$ moduli with cases where the cover moduli has dimension 1 . The gains come by detecting the moduli resemblances to, and differences from, modular curves.

We emphasize: Our technique produces a parameter for families of equations from an essential defining property of the equations. We aim for a direct description of that parameter using the defining property. These examples connect two themes useful for intricate work on families of equations. We refer to these as two genus 0 problems considered by John Thompson. Our first version is a naive form.

(1.1a) Problem $_{1}^{g=0}$ : If a moduli space of algebraic relations is a genus 0 curve, where can we find a uniformizer for it?

(1.1b) Problem ${ }_{2}^{g=0}$ : Excluding symmetric, alternating, cyclic and dihedral groups, what others are monodromy groups for primitive genus 0 covers?

Our later versions of each statement explicitly connect with well-known problems. [Fr80], [Fr99], [GMS03] show examples benefiting from the monodromy method. 
We use the latest Nielsen class techniques ( $\$ 2.2$; excluding the shift-incidence matrix from [BFr02, §9] and [FrS04]) to understand these parameter spaces as natural $j$-line covers. They are not modular curves, though emulating [BFr02] we observe modular curve-like properties.

Applying the Riemann-Roch Theorem to Problem ${ }_{1}^{g=0}$ does not actually answer the underlying question. Even when (say, from Riemann-Hurwitz) we find a curve has genus 0 that doesn't trivialize uniformizing its function field. Especially when the moduli space has genus 0: We seek a uniformizer defined by the moduli problem. That the $j$-line covers of our examples have genus 0 allows them to effectively parametrize (over a known field) solutions to problems with a considerable literature. We justify that — albeit, briefly — to give weight to our choices.

[FaK01] uses $k$-division $\theta$-nulls (from elliptic curves) to uniformize certain modular curves. Those functions, however, have nothing to do with the moduli for our examples. Higher dimensional $\theta$-nulls on the (1-dimensional) upper-half plane are akin to, but not the same as, what quadratic form people call $\theta$-functions. The former do appear in our examples of $\operatorname{Problem}_{1}^{g=0}(\S 7.2 .5$; we explain more there on this $\theta$-confusing point). We are new at Monstrous Moonshine, though the required expertise documented by [Ra00] shows we're not alone. Who can predict from where significant uniformizers will arise? If a $\theta$-null intrinsically attaches to the moduli problem, we'll use it.

1.2. Detailed results. $\$ 7.2 .3$ has the precise definition of 0 -sporadic (also, polynomial 0 -sporadic and the general $g$-sporadic). All modular curves appear as (reduced; see §2.2.2) families of genus 0 covers $[\mathbf{F r} 78, \S 2]$. Only, however, finitely many modular curves have genus 0 . Our first examples are moduli spaces for polynomial 0 -sporadic groups responding to (1.1b). These moduli spaces are genus 0 covers of the $j$-line, responding to (1.1a), yet they are not modular curves.

1.2.1. The moduli of three 0-sporadic monodromy groups. Three polynomial 0sporadic groups stand out on Müller's list (§2.4): These have degrees $n=7,13$ and 15, with four branch points (up to reduced equivalence $\$ 2.2 .2$ ). Their families have genus 0 suiting question (1.1a). Each case sums up in one (for each $n \in\{7,13,15\}$ ) genus $0 j$-line cover $\left(\psi_{n}: X_{n} \rightarrow \mathbb{P}_{j}^{1}\right)$ over $\mathbb{Q}$. We tell much about these spaces, their $b$-fine moduli properties and their cusps (Prop. 4.1 and Prop. 5.1).

We stress the uniqueness of $\psi_{n}$, and its $\mathbb{Q}$ structure. Reason: The moduli problem defining it does not produce polynomials over $\mathbb{Q}$. Let $K_{13}$ be the unique degree 4 extension of $\mathbb{Q}$ in $\mathbb{Q}\left(e^{2 \pi i / 13}\right)$. For $n=13$, a parameter uniformizing $X_{13}$ as a $\mathbb{Q}$ space gives coordinates for the four (reduced) families of polynomials over $K_{13}$. These appear as solutions of Davenport's problem (§2.1). Resolving Davenport's problem (combining group theory and arithmetic in [Fr73], [Fe80], [Fr80] and [Fr99]) suggested that genus 0 covers have a limited set of monodromy groups $\left(\right.$ Problem $\left._{2}^{g=0}\right)$. $[$ Fr99, $§ 5]$ and $[\mathbf{F r}$ 04, App. D] has more on the applications.

1.2.2. Modular curve-like genus 0 and 1 curves. Our second example is closer to classical modular curve themes, wherein uniformizers of certain genus 0 curves appear from $\theta$-functions. $\$ 7.2 .5$ briefly discusses Monstrous Moonshine for comparison. Our situation is the easiest rank 2 Modular Tower, defined by the group $F_{2} \times^{s} \mathbb{Z} / 3$, with $F_{2}$ a free group on two generators. We call this the $n=3$ case. For each prime $p \neq 3$, and for each integer $k \geq 0$, there is a map $\bar{\psi}_{p, k}: \overline{\mathcal{H}}_{p, k}^{\text {rd }} \rightarrow \mathbb{P}_{j}^{1}$ with $\overline{\mathcal{H}}_{p, k}^{\text {rd }}$ a (reduced) moduli space. The gist of Prop. 6.5: Each such $\overline{\mathcal{H}}_{p, k}^{\text {rd }}$ is nonempty 
(and $\bar{\psi}_{p, k}$ is a natural $j$-line cover). For a given $p$ the collection $\left\{\overline{\mathcal{H}}_{k, p}^{\text {rd }}\right\}_{k \geq 0}$ forms a projective system; we use this below.

We contrast the $n=3$ case with the case $F_{2} \times{ }^{s} \mathbb{Z} / 2$. This is the $n=2$ case: $-1 \in\{ \pm 1\}=\mathbb{Z} / 2$ maps generators of $F_{2}$ to their inverses. We do this to give the Modular Tower view of noncomplex multiplication in Serre's Open Image Theorem ([Se68, IV-20]). The gist of Prop. 6.3: Serre's Theorem covers less territory than might be expected. [Fr04, §5.2-5.3] applies this to producing genus 0 exceptional covers. This shows Davenport's problem is not an isolated example.

The (strong) Main Conjecture on Modular Towers [FrS04, §1.2] says the following for $n=3$. Only finitely many $\overline{\mathcal{H}}_{p, k}^{\text {rd }}$ s have a genus 0 or 1 (curve) component. These are moduli spaces, and rational points on such components interpret significantly for many problems. For $n=2$ the corresponding spaces are modular curves, and they have but one component. Known values of $(p, k)$ where $\overline{\mathcal{H}}_{p, k}^{\text {rd }}$ has more than one component include $p=2$, with $k=0$ and 1 . $\S 6.1$ explains the genus 0 and 1 components for the second of these. For $j$-line covers coming from Nielsen classes there is a map from elements in the Nielsen class to cusps. The most modular curve-like property of these spaces is that they fall in sequences attached to a prime $p$. Then, especially significant are the $g-p^{\prime}$ cusps ( $\left.\S 6.4\right)$.

Most studied of the $\mathrm{g}-p^{\prime}$ cusps are those we call Harbater-Mumford. For example, in this language the width $p$ (resp. 1) cusp on the modular curve $X_{0}(p)(p$ odd) is the Harbater-Mumford (resp. shift of a Harbater-Mumford) cusp (Ex. 6.6). Prob. 6.7 is a conjectural refinement of Prop. 6.3. This distinguishes those components containing H-M cusps among the collection of all components of $\overline{\mathcal{H}}_{p, k}^{\text {rd }} \mathrm{s}$.

If right, we can expect applications for those components that parallel [Se68] (for $n=2$ ). We conclude with connections between $\operatorname{Problem}_{1}^{g=0}$ and Problem ${ }_{2}^{g=0}$. This gives an historical context for using cusps of $j$-line covers from Nielsen classes.

(1.2a) Comparison of our computations with computer construction of equations for the Davenport pair families in [CoCa99] (§7.1).

(1.2b) The influence of John Thompson on Problem ${ }_{1}^{g=0}$ and $\operatorname{Problem}_{2}^{g=0}(\S 7.2)$.

\section{Examples from Problem ${ }_{2}^{g=0}$}

We briefly state Davenport's problem and review Nielsen classes. Then, we explain Davenport's problem's special place among polynomial 0-sporadic groups.

2.1. Review of Davenport's problem. The name Davenport pair (now called S(trong)DP) first referred to pairs $(f, g)$ of polynomials, over a number field $K$ (with ring of integers $\mathcal{O}_{K}$ ) satisfying this.

(2.1) Range equality: $f(\mathcal{O} / \boldsymbol{p})=g(\mathcal{O} / \boldsymbol{p})$ for almost all prime ideals $\boldsymbol{p}$ of $\mathcal{O}_{K}$. Davenport asked this question just for polynomials over $\mathbb{Q}$. We also assume there should be no linear change of variables (even over $\bar{K}$ ) equating the polynomials. This is an hypothesis that we intend from this point. There is a complete description of the Davenport pairs where $f$ is indecomposable $(\S 2.2)$.

In this case such polynomials are i(sovalent)DPs: Each value in the range of $f$ or $g$ is achieved with the same multiplicity by both polynomials. As in [AFH03, 7.30], this completely describes all such pairs even with a weaker hypothesis: $(2.1)$ holds for just $\infty$-ly many prime ideals of $\mathcal{O}_{K}$.

2.2. Review of Nielsen classes. A Nielsen class is a combinatorial invariant attached to a (ramified) cover $\varphi: X \rightarrow \mathbb{P}_{z}^{1}$ of compact Riemann surfaces. If 
$\operatorname{deg}(\varphi)=n$, let $G_{\varphi} \leq S_{n}$ be the monodromy group of $\varphi$. The cover is primitive or indecomposable if the following equivalent properties hold.

(2.2a) It has no decomposition $X \stackrel{\varphi^{\prime}}{\longrightarrow} W \stackrel{\varphi^{\prime \prime}}{\longrightarrow} \mathbb{P}_{z}^{1}$, with $\operatorname{deg}\left(\varphi^{\prime}\right) \geq 2, \operatorname{deg}\left(\varphi^{\prime \prime}\right) \geq 2$.

(2.2b) $G_{\varphi}$ is a primitive subgroup of $S_{n}$.

Let $\boldsymbol{z}$ be the branch points of $\varphi, U_{z}=\mathbb{P}_{z}^{1} \backslash\{\boldsymbol{z}\}$ and $z_{0} \in U_{z}$. Continue points over $z_{0}$ along paths based at $z_{0}$, having the following form: $\gamma \cdot \delta_{i} \gamma^{-1}, \gamma, \delta$ on $U_{\boldsymbol{z}}$ and $\delta_{i}$ a small clockwise circle around $z_{i}$. This attaches to $\varphi$ a collection of conjugacy classes $\mathbf{C}=\left(\mathrm{C}_{1}, \ldots, \mathrm{C}_{r}\right\}$, one for each $z_{i} \in \boldsymbol{z}$. The associated Nielsen class:

$$
\mathrm{Ni}=\mathrm{Ni}(G, \mathbf{C})=\left\{\boldsymbol{g}=\left(g_{1}, \ldots, g_{r}\right) \mid g_{1} \cdots g_{r}=1,\langle\boldsymbol{g}\rangle=G \text { and } \boldsymbol{g} \in \mathbf{C}\right\} .
$$

Product-one is the name for the condition $g_{1} \cdots g_{r}=1$. From it come invariants attached to spaces defined by Nielsen classes. Generation is the name of condition $\langle\boldsymbol{g}\rangle=G$. Writing $\boldsymbol{g} \in \mathbf{C}$ means the $g_{i}$ s define conjugacy classes in $G$, possibly in another order, the same (with multiplicity) as those in C. So, each cover $\varphi: X \rightarrow \mathbb{P}_{z}^{1}$ has a uniquely attached Nielsen class: $\varphi$ is in the Nielsen class $\operatorname{Ni}(G, \mathbf{C})$.

2.2.1. Standard equivalences. Suppose we have $r$ (branch) points $\boldsymbol{z}$, and a corresponding choice $\overline{\boldsymbol{g}}$ of classical generators for $\pi_{1}\left(U_{\boldsymbol{z}}, z_{0}\right)$ [BFr02, §1.2]. Then, $\operatorname{Ni}(G, \mathbf{C})$ lists all homomorphisms from $\pi_{1}\left(U_{\boldsymbol{z}}, z_{0}\right)$ to $G$. These give a cover with branch points $\boldsymbol{z}$ associated to $(G, \mathbf{C})$. Elements of $\mathrm{Ni}(G, \mathbf{C})$ are branch cycle descriptions for these covers relative to $\overline{\boldsymbol{g}}$. Equivalence classes of covers with a fixed set of branch points $z$, correspond one-one to equivalence classes on $\operatorname{Ni}(G, \mathbf{C})$. We caution: Attaching a Nielsen class representative to a cover requires picking one from many possible $r$-tuples $\overline{\boldsymbol{g}}$. So, it is not an algebraic process.

[BFr02, §3.1] reviews common equivalences with examples and relevant definitions. such as the group $\mathcal{Q}^{\prime \prime}$ below. Let $N_{S_{n}}(G, \mathbf{C})$ be those $g \in S_{n}$ normalizing $G$ and permuting the collection of conjugacy classes in C. Absolute (resp. inner) equivalence classes of covers (with branch points at $\boldsymbol{z}$ ) correspond to the elements of $\operatorname{Ni}(G, \mathbf{C}) / N_{S_{n}}(G, \mathbf{C})=\operatorname{Ni}(G, \mathbf{C})^{\text {abs }}$ (resp. $\left.\mathrm{Ni}(G, \mathbf{C}) / G=\operatorname{Ni}(G, \mathbf{C})^{\text {in }}\right)$. Especially in $\S 3$ we use absolute, inner and for each of these reduced equivalence. These show how to compute specific properties of $\mathcal{H}(G, \mathbf{C})^{\text {abs }}, \mathcal{H}(G, \mathbf{C})^{\text {in }}$ and their reduced versions, parametrizing the equivalences classes of covers as $\boldsymbol{z}$ varies.

2.2.2. Reduced Nielsen classes. Reduced equivalence corresponds each cover $\varphi: X \rightarrow \mathbb{P}_{z}^{1}$ to $\alpha \circ \varphi: X \rightarrow \mathbb{P}_{z}^{1}$, running over $\alpha \in \mathrm{PGL}_{2}(\mathbb{C})$. If $r=4$, a nontrivial equivalence arises because for any $z$ there is a Klein 4-group in $\mathrm{PGL}_{2}(\mathbb{C})$ mapping $z$ into itself. (An even larger group leaves special, elliptic, $z$ fixed.) This interprets as an equivalence from a Klein 4-group $\mathcal{Q}^{\prime \prime}$ acting on Nielsen classes (§4). Denote associated absolute (resp. inner) reduced Nielsen class representatives by

$\mathrm{Ni}(G, \mathbf{C}) /\left\langle N_{S_{n}}(G, \mathbf{C}), \mathcal{Q}^{\prime \prime}\right\rangle=\operatorname{Ni}(G, \mathbf{C})^{\text {abs,rd }}\left(\operatorname{resp} . \operatorname{Ni}(G, \mathbf{C}) /\left\langle G, \mathcal{Q}^{\prime \prime}\right\rangle=\mathrm{Ni}(G, \mathbf{C})^{\text {in,rd }}\right)$.

These give formulas for branch cycles presenting $\mathcal{H}(G, \mathbf{C})^{\text {abs,rd }}$ and $\mathcal{H}(G, \mathbf{C})^{\text {in,rd }}$ as upper half plane quotients by a finite index subgroup of $\mathrm{PSL}_{2}(\mathbb{Z})$. This is a ramified cover of the classical $j$-line branching over the traditional places (normalized in [BFr02, Prop. 4.4] to $j=0,1, \infty)$. Points over $\infty$ are meaningfully called cusps. Here is an example of how we will use these.

$\S 4$ computes from these tools two $j$-line covers (dessins d'enfant) conjugate over $\mathbb{Q}(\sqrt{-7})$ parametrizing reduced classes of degree 7 Davenport polynomial pairs. In fact, the (by hand) Nielsen class computations show the covers are equivalent over $\mathbb{Q}$. This same phenomenon happens for all pertinent degrees $n=7,13,15$, though 
the field $\mathbb{Q}(\sqrt{-7})$ changes and corresponding Nielsen classes have subtle differences. You can see these by comparing $n=7$ with $n=13(\S 5)$.

2.3. Davenport Pair monodromy groups. Let $u \geq 2$. A Singer cycle is a generator $\alpha$ of $\mathbb{F}_{q^{u+1}}^{*}$, acting by multiplication as a matrix through identifying $\mathbb{F}_{q}^{u+1}$ and $\mathbb{F}_{q^{u+1}}$. Its image in $\mathrm{PGL}_{u+1}$ acts on points and hyperplanes of $\mathbb{P}^{u}\left(\mathbb{F}_{q}\right)$.

Let $G$ be a group with two doubly transitive representations $T_{1}$ and $T_{2}$, equivalent as group representations, yet not permutation equivalent, and with $g_{\infty} \in G$ an $n$-cycle in $T_{i}, i=1,2$. Excluding the well-documented degree 11 case, $G$ has these properties $([\mathbf{F e} 80],[\mathbf{F r} \mathbf{8 0}],[\mathbf{F r} \mathbf{9 9}, \S 8])$ with $\mathrm{C}_{\alpha}$ the conjugacy class of $g_{\infty}$.

(2.3a) $G \geq \mathrm{PSL}_{u+1}\left(\mathbb{F}_{q}\right) ; T_{1}$ and $T_{2}$ act on points and hyperplanes of $\mathbb{P}^{u}$.

(2.3b) $n=\left(q^{u+1}-1\right)(q-1)$ and $g_{\infty}$ is a Singer $n$-cycle.

2.3.1. Difference sets. Here is how difference sets (§3.2) appear from (2.3).

Definition 2.1. Call $\mathcal{D} \leq \mathbb{Z} / n$ a difference set if nonzero differences from $\mathcal{D}$ distribute evenly over $\mathbb{Z} \backslash\{0\}$. The multiplicity $v$ of the appearance of each element is the multiplicity of $\mathcal{D}$. Regard a difference set and any translate of it as equivalent.

Given the linear representation from $T_{1}$ on $x_{1}, \ldots, x_{n}$, the representation $T_{2}$ is on $\left\{\sum_{i \in \mathcal{D}+j} x_{i}\right\}_{j=1}^{n}$ with $\mathcal{D}$ a difference set. The multiplier group $M_{n}$ of $\mathcal{D}$ is

$$
\left\{m \in(\mathbb{Z} / n)^{*} \mid m \cdot \mathcal{D}=\mathcal{D}+j_{m}, \text { with } j_{m} \in \mathbb{Z} / n\right\} .
$$

We say $m \cdot \mathcal{D}$ is equivalent to $\mathcal{D}$ if $m \in M_{n}$. In $\operatorname{PGL}_{u+1}\left(\mathbb{F}_{q}\right), \alpha^{m}$ is conjugate to $\alpha$ exactly when $m \in M_{n}$. The difference set $-\mathcal{D}$ corresponds to an interchange between the representations on points and hyperplanes. Conjugacy classes in $\operatorname{PGL}_{u+1}\left(\mathbb{F}_{q}\right)$ of powers of $\alpha$ correspond one-one to difference sets equivalence classes $\bmod n$.

2.3.2. Davenport pair Nielsen classes. We label our families of polynomials by an $m \in(\mathbb{Z} / n)^{*} \backslash M_{n}$ that multiplies the difference set to an inequivalent difference set. Our families are of absolute reduced classes of covers in a Nielsen class. Conjugacy classes have the form $\left(\mathrm{C}_{1}, \ldots, \mathrm{C}_{r-1}, \mathrm{C}_{\alpha}\right)$, the groups satisfy $G \geq \mathrm{PSL}_{u+1}\left(\mathbb{F}_{q}\right)$, and covers in the class have genus 0 . Two results of Feit show $r-1 \leq 3$ :

(2.4a) ind $(\mathrm{C}) \geq n / 2$ if $\mathrm{C}$ is a conjugacy classes of $\mathrm{PGL}_{u+1}\left(\mathbb{F}_{q}\right)[\mathbf{F e} 73]$.

$(2.4 \mathrm{~b})$ ind $(\mathrm{C}) \geq n(q-1) / q$ if $\mathrm{C}$ is a conjugacy class in $\mathrm{P} \Gamma \mathrm{L}_{u+1}\left(\mathbb{F}_{q}\right)[\mathbf{F e}$ 92].

. Conclude: If $n$ is odd, then $r-1=3$ implies the following for respective cases.

(2.5a) $n=7: \mathrm{C}_{i} \mathrm{~s}$ are in the conjugacy class of transvections (fixing a hyperplane), with index 2 . So, they are all conjugate.

(2.5b) $n=13: \mathrm{C}_{i} s$ are in the conjugacy class of elements fixing a a hyperplane (determinant -1 ), so they generate $\mathrm{PGL}_{3}(\mathbb{Z} / 3)$ and all are conjugate.

(2.5c) $n=15$ : Two of the $\mathrm{C}_{i} \mathrm{~s}$ are in the conjugacy class of transvections, and one fixes just a line.

(2.5d) $n \neq 7,13,15: r=3$.

Transvections in $\mathrm{GL}_{u+1}$ have the form $\boldsymbol{v} \mapsto \boldsymbol{v}+\mu_{H}(\boldsymbol{v}) \boldsymbol{v}_{0}$ with $\mu_{H}$ a linear functional with kernel a hyperplane $H$, and $\boldsymbol{v}_{0} \in H \backslash\{0\}$ [A57, p. 160]. For $q$ a power of two, these are involutions: exactly those fixing points of a hyperplane. For $q$ odd, involutions fixing the points of a hyperplane (example, induced by a reflection in $\mathrm{GL}_{n+1}$ in the hyperplane) have the maximal number of fixed points. When $q$ is a power of 2 , there are involutions fixing precisely one line. Jordan normal form 
shows these are conjugate to

$$
\left(\begin{array}{llll}
1 & 0 & 0 & 0 \\
a & 1 & 0 & 0 \\
0 & b & 1 & 0 \\
0 & 0 & c & 1
\end{array}\right) .
$$

This is an involution if and only if $a b=b c=0$. So, either $b=0$ or $a=c=0$. Only in the latter case is the fixed space a line. So, given the conjugacy class of $g_{\infty}$, only one possible Nielsen class defines polynomial Davenport pairs when $n=15$.

2.4. Müller's list of primitive polynomial monodromy groups. We reprise Müller's list of the polynomial 0-sporadic groups ([Mu95]). Since such a group comes from a primitive cover, it goes with a primitive permutation representation. As in $\S 7.2 .3$ we regard two inequivalent representations of the same group as different 0-sporadic groups. We emphasize how pertinent was Davenport's problem. Exclude (finitely many) groups with simple core $\mathrm{PSL}_{2}\left(\mathbb{F}_{q}\right)$ (for very small $q$ ) and the Matthieu groups of degree 11 and 23. Then, all remaining groups from his list are from $[\mathbf{F r} 73]$ and have properties $(2.3)$. [Fr99, $§ 9]$ reviews and completes this. These six polynomial 0-sporadic groups (with corresponding Nielsen classes) all give Davenport pairs. We concentrate on those three having one extra property:

(2.6) Modulo $\mathrm{PGL}_{2}(\mathbb{C})$ (reduced equivalence as in $\S 2.2 .2$ ) action, the space of these polynomials has dimension at least (in all cases, equal) 1 .

We restate the properties shown above for these polynomial covers.

- They have degrees from $\{7,13,15\}$ and $r=4$.

- All $r \geq 4$ branch point indecomposable polynomial maps in an iDP pair (§2) are in one of the respectively, 2, 4 or 2 Nielsen classes corresponding to the respective degrees $7,13,15$.

[Fr73] outlines this. [Fr80, §2.B] uses it to explain Hurwitz monodromy action.

Let $\mathcal{H}_{7}^{\mathrm{DP}}, \mathcal{H}_{13}^{\mathrm{DP}}$ and $\mathcal{H}_{15}^{\mathrm{DP}}$ denote the spaces of polynomial covers that are one from a Davenport pair having four branch points (counting $\infty$ ). The subscript decoration corresponds to the respective degrees. We assume absolute, reduced equivalence (as in $\S 2.2 .2$ ).

\section{Explanation of the components for $\mathcal{H}_{7}^{\mathrm{DP}}, \mathcal{H}_{13}^{\mathrm{DP}}, \mathcal{H}_{15}^{\mathrm{DP}}$}

The analytic families of respective degree $n$ polynomials fall into several components. Each component, however, corresponds to a different Nielsen class. For example, $\mathcal{H}_{7}^{\mathrm{DP}}$, the space of degree 7 Davenport polynomials has two components: with a polynomial associated to a polynomial in the other as a Davenport Pair.

3.1. Explicit difference sets. Often we apply Nielsen classes to problems about the realization of covers over $\mathbb{Q}$. Then, one must assume $\mathbf{C}$ is rational. [Fr73, Thm. 2] proved (free of the finite simple group classification) that no indecomposable polynomial DPs could occur over $\mathbb{Q}$.

There are polynomial covers in our Nielsen classes. So, $\boldsymbol{g} \in \mathrm{Ni}(G, \mathbf{C})$ has an $n$-cycle entry, $g_{\infty}$. These conjugacy classes for all $n$ are similar:

$$
\mathbf{C}_{n, u ; k_{1}, k_{2}, k_{3}}=\left(\mathrm{C}_{2^{k_{1}}}, \mathrm{C}_{2^{k_{2}}}, \mathrm{C}_{2^{k_{3}}}, \mathrm{C}_{(\alpha)^{u}}\right) \text {, }
$$

where $\mathrm{C}_{2^{k}}$ denotes a (nontrivial) conjugacy class of involutions of index $k$ and $(u, n)=1$. We explain the case $n=7$ in the following rubric. 
(3.1a) Why $\mathrm{C}_{2^{k_{i}}}=\mathrm{C}_{2^{2}}, j=1,2,3$, is the conjugacy class of a transvection (denote the resulting conjugacy classes by $\mathbf{C}_{7, u ; 3 \cdot 2^{2}}$ ).

(3.1b) Why the two components of $\mathcal{H}_{7}^{\mathrm{DP}}$ are

$$
\mathcal{H}_{+}=\mathcal{H}\left(\mathrm{PSL}_{3}(\mathbb{Z} / 2), \mathbf{C}_{7,1 ; 3 \cdot 2^{2}}\right)^{\text {abs }, \mathrm{rd}} \text { and } \mathcal{H}_{-}=\mathcal{H}\left(\mathrm{PSL}_{3}(\mathbb{Z} / 2), \mathbf{C}_{7,-1 ; 3 \cdot 2^{2}}\right)^{\text {abs,rd }} .
$$

(3.1c) Why the closures of $\mathcal{H}_{ \pm}$over $\mathbb{P}_{j}^{1}$ (as natural $j$-line covers) are equivalent genus 0 , degree 7 covers over $\mathbb{Q}$.

(3.1d) Why $\mathcal{H}_{ \pm}$, as degree 7 Davenport moduli, have definition field $\mathbb{Q}(\sqrt{-7})$.

3.2. Difference sets give properties (3.1a) and (3.1b). [Fr73, Lem. 4] normalizes Nielsen class representatives $\left(g_{1}, g_{2}, g_{3}, g_{\infty}\right)$ for DP covers so that in both representations $T_{j, n}, j=1,2, g_{\infty}=(12 \ldots n)^{-1}$ identifies with some allowable $\alpha_{n}$.

Regard $\left(g_{\infty}\right) T_{1, n}\left(g_{\infty}\right.$ in the representation $\left.T_{1, n}\right)$ as translation by -1 on $\mathbb{Z} / n$. Then, $\left(g_{\infty}\right) T_{2, n}$ is translation by -1 on the collection of sets $\{\mathcal{D}+c\}_{c \in \mathbb{Z} / n}$. Take $v$ to be the multiplicity of $\mathcal{D}$. Then, $v(n-1)=k(k-1)$ with $1<k=|\mathcal{D}|<n-1$. In $\mathrm{PGL}_{u+1}\left(\mathbb{F}_{q}\right), \alpha_{n}^{u}$ is conjugate to $\alpha_{n}$ if and only if $u \cdot \mathcal{D}$ is a translation of $\mathcal{D}$. That is, $u$ is a multiplier of the design. Also, -1 is always a nonmultiplier [Fr73, Lem. 5]. Here $n=7$, so $v(n-1)=k(k-1)$ implies $k=3$ and $v=1$. You find $\bmod 7$ : $\mathcal{D}=\{1,2,4\}$ and $-\mathcal{D}$ are the only difference sets mod translation. The multiplier of $\mathcal{D}$ is $M_{7}=\langle 2\rangle \leq(\mathbb{Z} / 7)^{*}: 2 \cdot \mathcal{D}=\mathcal{D}+c(c=0$ here $)$.

Covers in this Nielsen class have genus 0. Now use that in $\operatorname{PSL}_{3}(\mathbb{Z} / 2)$, (acting on points) the minimal possible index is 2 . We labeled the transvection conjugacy class achieving that as $\mathrm{C}_{2^{2}}$. So, 2 is the index of entries of $\boldsymbol{g}$ for all finite branch points. We have shown (3.1b) has the only two possible Nielsen classes.

3.3. Completing property (3.1b). We now show the two spaces $\mathcal{H}_{ \pm}$are irreducible, completing property $(3.1 \mathrm{~b})$.

Computations in [Fr95a, p. 349] list absolute Nielsen class representatives with $g_{\infty}$ the 4 th entry. Label finite branch cycles $\left(g_{1}, g_{2}, g_{3}\right)$ (corresponding to a polynomial cover, having $g_{\infty}$ in fourth position) as $Y_{1}, \ldots, Y_{7}$. There are 7 up to conjugation by $\left\langle g_{\infty}\right\rangle$, the only allowance left for absolute equivalence.

$$
\begin{aligned}
& Y_{1}:\left((35)(67),((45)(62),(36)(12)) ; Y_{2}:((35)(67),(36)(12),(31)(45)) ;\right. \\
& Y_{3}:((35)(67),(16)(23),(45)(62)) ; Y_{4}:((35)(67),(13)(45),(23)(16)) ; \\
& Y_{5}:((37)(56),(13)(45),(23)(47)) ; Y_{6}:((37)(56),(23)(47),(12)(75)) ; \\
& Y_{7}:((37)(56),((12)(75),(13)(45)))
\end{aligned}
$$

The element (35)(67) represents a transvection fixing points of a line $\Longleftrightarrow$ elements of $\mathcal{D}$. Note: All entries in $Y_{1}, \ldots, Y_{7}$ of Table (3.2) correspond to transvections. So these are conjugate to $(35)(67)$. From this point everything reverts to Hurwitz monodromy calculation with The elements $q_{i}, i=1,2,3$. Each acts by a twisting action on any 4-tuple representing a Nielsen class element. For example,

$$
q_{2}: \boldsymbol{g} \mapsto(\boldsymbol{g}) q_{2}=\left(g_{1}, g_{2} g_{3} g_{2}^{-1}, g_{2}, g_{4}\right) .
$$

3.4. The analog for $n=13$ and 15. Up to translation there are 4 difference sets modulo 13. All cases are similar. So we choose $\mathcal{D}_{13}=\{1,2,4,10\}$ to be specific. Others come from multiplications by elements of $(\mathbb{Z} / 13)^{*}$. Multiplying by $\langle 3\rangle=M_{13}(\S 2.3)$ preserves this difference set (up to translation).

Each of $g_{1}, g_{2}, g_{3}$ fixes all points of some line, and one extra point, a total of five points. Any column matrix $A=\left(\boldsymbol{v}\left|\boldsymbol{e}_{2}\right| \boldsymbol{e}_{2}\right)$ with $\boldsymbol{v}$ anything, and $\left\{\boldsymbol{e}_{i}\right\}_{i=1}^{3}$ the 
standard basis of $\mathbb{F}_{3}^{3}$, fixes all points of the plane $P$ of vectors with 0 in the 1 st position. Stipulate one other fixed point in $\mathbb{P}^{2}\left(\mathbb{F}_{3}\right)$ to determine $A$ in $\mathrm{PGL}_{3}\left(\mathbb{F}_{3}\right)$.

Let $\zeta_{13}=e^{2 \pi i / 13}$. Identify $G\left(\mathbb{Q}\left(\zeta_{13}\right) / \mathbb{Q}\right)$ with $(\mathbb{Z} /(13))^{*}$. Let $K_{13}$ be the fixed field of $M_{13}$ in $\mathbb{Q}\left(\zeta_{13}\right)$. Therefore $K_{13}$ is $\mathbb{Q}\left(\zeta_{13}+\zeta_{13}^{3}+\zeta_{13}^{9}\right)$, a degree 4 extension of $\mathbb{Q}$. Akin to when $n=7$ take $g_{\infty}=(12 \ldots 1213)^{-1}$. The distinct difference sets (inequivalent under translation) appear as $6^{j} \cdot \mathcal{D}_{13}, j=0,1,2,3$ (6 generates the order 4 cyclic subgroup of $\left.(\mathbb{Z} / 13)^{*}\right)$.

For future reference, though we don't do the case $n=15$ completely here, $\mathcal{D}_{15}=\{0,5,7,10,11,13,14\}$ is a difference set $\bmod 15$. Its multiplicity is $v$ in $v(15-1)=k(k-1)$ forcing $k=7$ and $v=3$. The multiplier group is $M_{15}=\langle 2\rangle$, so the minimal field of definition of polynomials in the corresponding Davenport pairs is $\mathbb{Q}\left(\sum_{j=0}^{3} \zeta_{15}^{2^{j}}\right)$, the degree 2 extension of $\mathbb{Q}$ that $\frac{1+\sqrt{-15}}{2}$ generates. So, this case, like $n=7$, has two families of polynomials appear as associated Davenport pairs.

As with $n=7$, use the notation $\mathrm{C}_{(\alpha)}, u \in(\mathbb{Z} / 13)^{*}$ for the conjugacy classes of powers of 13-cycles. Prop. 5.1 shows the following.

(3.4a) Why $\mathrm{C}_{2^{k_{i}}}=\mathrm{C}_{2^{4}}, i=1,2,3$, is the conjugacy class fixing all points of a plane (denote the resulting conjugacy classes by $\mathbf{C}_{13, u ; 3 \cdot 2^{4}}$ ).

(3.4b) Why the four components of $\mathcal{H}_{13}^{\text {DP }}$ are

$$
\mathcal{H}_{i}=\mathcal{H}\left(\mathrm{PGL}_{3}(\mathbb{Z} / 3), \mathbf{C}_{13,6^{i} ; 3 \cdot 2^{4}}\right)^{\text {abs }, \mathrm{rd}}, i=0,1,2,3 .
$$

(3.4c) Why closure of all $\mathcal{H}_{i}$ s over $\mathbb{P}_{j}^{1}$ are equivalent genus 0 , degree 13 covers over $\mathbb{Q}$. Yet, $\mathcal{H}_{i}$, as degree 13 Davenport moduli, has definition field $K_{13}$.

\section{4. $j$-line covers for polynomial $\mathrm{PGL}_{3}(\mathbb{Z} / 2)$ monodromy}

We produce branch cycles for the two $j$-line covers $\bar{\psi}_{ \pm}: \overline{\mathcal{H}}_{ \pm} \rightarrow \mathbb{P}_{j}^{1}, i=1,2$, parametrizing degree 7 Davenport (polynomial) pairs. Prop. 4.1 says they are equivalent as $j$-line covers, though distinct as families of degree 7 covers.

4.1. Branch cycle presentation and definition field. Our original notation, $\mathcal{H}_{ \pm}$is for points of $\overline{\mathcal{H}}_{ \pm}$not lying over $j=\infty$. Each $\boldsymbol{p}_{+} \in \mathcal{H}_{+}(\overline{\mathbb{Q}})$ has a corresponding point $\boldsymbol{p}_{-} \in \mathcal{H}_{-}(\overline{\mathbb{Q}})$ denoting a collection of polynomial pairs

$$
\left\{\left(\beta \circ f_{\boldsymbol{p}_{+}}, \beta \circ f_{\boldsymbol{p}_{-}}\right)\right\}_{\beta \in \mathrm{PGL}_{2}(\overline{\mathbb{Q}})} \text {. }
$$

The absolute Galois group of $\mathbb{Q}\left(\boldsymbol{p}_{+}\right)=\mathbb{Q}\left(\boldsymbol{p}_{-}\right)$maps this set into itself. Representatives for absolute Nielsen class elements in Table (3.2) suffice for our calculation. This is because reduced equivalence adds the action of $\mathcal{Q}^{\prime \prime}=\left\langle\left(q_{1} q_{2} q_{3}\right)^{2}, q_{1} q_{3}^{-1}\right\rangle$. This has the following effect.

(4.1) Each $\boldsymbol{g} \in \mathrm{Ni}_{+}$is reduced equivalent to a unique absolute Nielsen class representative with $g_{\infty}$ in the 4 th position.

Example: If $\boldsymbol{g}$ has $g_{\infty}$ in the 3 rd position, apply $q_{1}^{-1} q_{3}$ to put it in the 4 th position.

[BFr02, Prop. 4.4] produces branch cycles for $\bar{\psi}_{ \pm}$. Reminder: The images of $\gamma_{0}=q_{1} q_{2}$ and $\gamma_{1}=q_{1} q_{2} q_{3}$ in $\left\langle q_{1}, q_{2}, q_{3}\right\rangle / \mathcal{Q}^{\prime \prime}=\mathrm{PSL}_{2}(\mathbb{Z})$ identify with canonical generators of respective orders 3 and 2 . The product-one condition $\gamma_{0} \gamma_{1} \gamma_{\infty}=1$ with $\gamma_{\infty}=q_{2}$ holds $\bmod \mathcal{Q}^{\prime \prime}$. Compute that $q_{1}$ with (3.3) action is $q_{1}^{*}=(351)(4762)$; $q_{2}$ acts as $q_{2}^{*}=(1342)(576)$. So, $\gamma_{0}$ acts as $\gamma_{0}^{*}=(375)(146)$. From product-one, $\gamma_{1}$ acts as $\gamma_{1}^{*}=(36)(71)(42)$.

Denote the respective conjugacy classes of $\left(\gamma_{0}^{*}, \gamma_{1}^{*}, \gamma_{\infty}^{*}\right)=\gamma^{*}$ in the group they generate by $\mathbf{C}^{*}=\left(\mathrm{C}_{0}, \mathrm{C}_{1}, \mathrm{C}_{\infty}\right)$. Denote $\mathbb{P}_{j}^{1} \backslash\{\infty\}$ by $U_{\infty}$. 
Proposition 4.1. The group $G=\left\langle\gamma_{0}^{*}, \gamma_{1}^{*}, \gamma_{\infty}^{*}\right\rangle$ is $S_{7}$. Then, $\gamma^{*}$ represents the only element in $\mathrm{Ni}\left(S_{7}, \mathbf{C}^{*}\right)^{\prime}$ : absolute equivalence classes with entries, in order, in the conjugacy class $\mathbf{C}^{*}$. So, there is a unique cover $\bar{\psi}_{7}: X_{7}=X \rightarrow \mathbb{P}_{j}^{1}$ in $\operatorname{Ni}\left(S_{7}, \mathbf{C}^{*}\right)^{\prime}$ (ramified over $\{0,1, \infty\}$ ). Restricting over $U_{\infty}$ gives $\psi_{7}: X^{\infty} \rightarrow U_{\infty}$ equivalent to $\mathcal{H}_{ \pm} \rightarrow U_{\infty}$ of (3.1b): It parametrizes each of the two absolute reduced families of $\mathbb{P}_{z}^{1}$ covers representing degree 7 polynomials that appear in a DP.

The projective curve $X$ has genus 0 . It is not a modular curve. The spaces $\mathcal{H}_{ \pm}$ are b-fine (but not fine; §4.2) moduli spaces over $\mathbb{Q}(\sqrt{-7})$. Their corresponding Hurwitz spaces are fine moduli spaces with a dense set of $\mathbb{Q}(\sqrt{-7})$ points. As a cover, however, $\bar{\psi}_{7}$ has definition field $\mathbb{Q}$, and $X$ has a dense set of $\mathbb{Q}$ points.

Proof. Since the group $G$ is transitive of degree 7 , it is automatically primitive. Further, $\left(\gamma_{\infty}^{*}\right)^{4}$ is a 3 -cycle. It is well-known that a primitive subgroup of $S_{n}$ containing a 3-cycle is either $A_{n}$ or $S_{n}$. In, however, our case $\gamma_{\infty}^{*} \notin A_{7}$, so $G=S_{7}$.

We outline why $\operatorname{Ni}\left(S_{7}, \mathbf{C}^{*}\right)^{\prime}$ has but one element. The centralizer of $\gamma_{\infty}^{*}$ is $U=\langle(1342),(576)\rangle$. Modulo absolute equivalence, any $\left(g_{0}, g_{1}, g_{\infty}\right) \in \operatorname{Ni}\left(S_{7}, \mathbf{C}^{*}\right)^{\prime}$ has $\gamma_{\infty}^{*}$ in the 3rd position. Let $F=\{2,5\}$ and let $x_{i}$ be the fixed element of $g_{i}$, $i=0,1$. Elements of $F$ represent the two orbits $O_{1}, O_{2}$ of $\gamma_{\infty}^{*}\left(2 \in O_{1}\right.$ and $\left.5 \in O_{2}\right)$. Conjugating by elements of $U$ gives four possibilities:

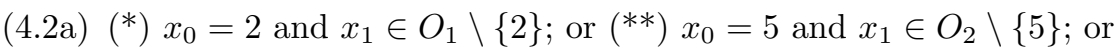

$(4.2 \mathrm{~b})\left(^{*}\right) x_{0}=5$ and $x_{1}=2$; or $\left(^{* *}\right) x_{0}=2$ and $x_{1}=5$.

We show the only possibility is $(4.2 \mathrm{~b})(* *)$, and for that, there is but one element. First we eliminate $(4.2 \mathrm{a})\left({ }^{*}\right)$ and $(* *)$. For $(4.2 \mathrm{a})(* *)$, then $x_{0}=5$ and $x_{1}=7$ or 6 . The former forces (up to conjugation by $U$ )

$$
g_{0}=(761) \cdots, g_{1}=(56) \cdots .
$$

Then, $g_{1} \gamma_{\infty}^{*}$ fixes 6 , contradicting $(6) g_{0}=1$. Also, $x_{1}=6$ fails. Consider (4.2a) $\left(^{*}\right)$, so $x_{1}=1$ or $3\left((24)\right.$ appears in $g_{0}$ automatically). Symmetry between the cases allows showing only $x_{1}=1$. This forces (143) in $g_{0}$ and $\left\langle\gamma_{\infty}^{*}, g_{0}\right\rangle$ is not transitive.

Now we eliminate $(4.2 \mathrm{~b})(*)$. Suppose $x_{0}=5, x_{1}=2$. This forces either

$$
g_{0}=(612) \cdots, g_{1}=(56)(17) \cdots \text { or } g_{0}=(6 x ?)(12 y), g_{1}=(56)(x 7)(y 4) \cdots .
$$

In the first, $4 \mapsto 2 \mapsto 6 \rightarrow 5$ in the product, so it doesn't work. By inspection, no value works for $y$ in the latter.

Conclude $x_{0}=2, x_{1}=5$. Previous analysis now produces but one possible element in $\operatorname{Ni}\left(S_{7}, \mathbf{C}^{*}\right)^{\prime}$. Applying Riemann-Hurwitz, using the index contributions of $g_{0}^{*}, g_{1}^{*}, g_{\infty}^{*}$ in order, the genus of the cover as $g_{7}$ satisfies $2\left(7+g_{7}-1\right)=3+4+5$. So, $g_{7}=0$.

That $X$ is not a modular curve follows from WohlFahrt's's Theorem [Wo64]. If it were, then its geometric monodromy group would be a quotient of $\mathrm{PSL}_{2}(\mathbb{Z} / N)$ : $N$ is the least common multiple of the cusp widths. So, $\operatorname{ord}\left(\gamma_{\infty}^{*}\right)=N=12$. Since, however, $\mathrm{PSL}_{2}(\mathbb{Z} / 12)=\mathrm{PSL}_{2}(3) \times \mathrm{PSL}_{2}(4)$, it does not have $S_{7}$ as a quotient.

We account for the b-fine moduli property. This is equivalent to $\mathcal{Q}^{\prime \prime}$ acting faithfully on absolute Nielsen classes [BFr02, Prop. 4.7]. This is so from (4.1), nontrivial elements of $\mathcal{Q}^{\prime \prime}$ move the conjugacy class of the 7-cycle. [BFr02, Prop. 4.7] also shows it is a fine reduced moduli space if and only if $\gamma_{0}^{*}$ and $\gamma_{1}^{*}$ have no fixed points. Here both have fixed points. So, the spaces $\mathcal{H}_{ \pm}$are not fine moduli spaces. 
4.2. b-fine moduli property. We explain [BFr02, §4.3.1] for our Davenport polynomial spaces. For the arithmetician, $\mathcal{P}$ having fine moduli over a field $K$ has this effect. For $\boldsymbol{p} \in \mathcal{P}$ corresponding to a specific algebraic object up to isomorphism, you have a representing object with equations over $K(\boldsymbol{p})$ with $K$. (We tacitly assume $\mathcal{P}$ is quasiprojective - our $\mathcal{H}$ and $\mathcal{H}^{\text {rd }}$ spaces are actually affine - to give meaning to the field generated by the coordinates of a point.)

4.2.1. The meaning of $b$-fine. The $\mathrm{b}$ in b-fine stands for birational. It means that if $\boldsymbol{p} \in \mathcal{H}^{\text {rd }}$ is not over $j=0$ or 1 , the interpretation above for $\boldsymbol{p}$ as a fine moduli point applies. It may apply if $j=0$ or 1 , though we cannot guarantee it.

If $\mathcal{P}$ is only a moduli space (not fine), then $\boldsymbol{p} \in \mathcal{P}$ may have no representing object over $K(\boldsymbol{p})$ (and certainly can't have one over a proper subfield of $K(\boldsymbol{p})$ ). Still, $G_{K(\boldsymbol{p})}$ stabilizes the complete set of objects over $\overline{K(\boldsymbol{p})}$ representing $\boldsymbol{p}$.

For any Nielsen class of four branch point covers, suppose the absolute (not reduced) space $\mathcal{H}$ has fine moduli. The condition for that is no element of $S_{n}$ centralizes $G$. That holds automatically for any primitive non-cyclic group $G \leq S_{n}$ (so in our cases; all references on rigidity in any form have this). For $\boldsymbol{p}$, denote the set of points described as the image of $\boldsymbol{p}$ in $U_{4}=\left(\left(\mathbb{P}_{z}^{1}\right)^{4} \backslash \Delta_{4}\right) / S_{4}$ by $z_{p}$. Assume $K$ is the field of rationality of the conjugacy classes. Conclude: There is a representative cover $\varphi_{\boldsymbol{p}}: X_{\boldsymbol{p}} \rightarrow \mathbb{P}_{z}^{1}$ branched over $\boldsymbol{z}_{\boldsymbol{p}}$ and having definition field $K(\boldsymbol{p})$.

4.2.2. Producing covers from the $b$-fine moduli property. Use the notation $\mathcal{H}^{\text {abs }}$ for nonreduced space representing points of the Nielsen class. Then $\mathcal{H}_{ \pm}$identifies with $\mathcal{H}_{ \pm}^{\text {abs }} / \mathrm{PGL}_{2}(\mathbb{C})$. Consider our degree 7 Davenport pair problem and their reduced spaces. The Nielsen classes are rational over $K=\mathbb{Q}(\sqrt{-7})$ (Prop. 4.1). Any $\boldsymbol{p}^{\text {rd }} \in \mathcal{H}_{ \pm}$represents a cover $\varphi_{\boldsymbol{p}^{\mathrm{rd}}}: X_{\boldsymbol{p}^{\mathrm{rd}}} \rightarrow Y_{\boldsymbol{p}^{\mathrm{rd}}}$ with $Y_{\boldsymbol{p}^{\mathrm{rd}}}$ a conic in $\mathbb{P}^{2}[\mathbf{B F r 0 2}$, Prop. 4.7] and $\varphi_{p^{\text {rd }}}$ degree 7. Often, even for b-fine moduli and $\boldsymbol{p}^{\text {rd }}$ not over 0 or 1 , there may be no $\boldsymbol{p} \in \mathcal{H}^{\text {abs }}$ lying over $\boldsymbol{p}^{\text {rd }}$ with $K(\boldsymbol{p})=K\left(\boldsymbol{p}^{\text {rd }}\right)$. Such a $\boldsymbol{p}$ would give $\psi_{\boldsymbol{p}}: X_{\boldsymbol{p}} \rightarrow \mathbb{P}_{z}^{1}$ over $K\left(\boldsymbol{p}^{\mathrm{rd}}\right)$ representing $\varphi_{\boldsymbol{p}^{\mathrm{rd}}}$.

Yet, in our special case, $X_{p^{\text {rd }}}$ has a unique point totally ramified over $Y_{p^{\text {rd }}}$. Its image in $Y_{\boldsymbol{p}^{\mathrm{rd}}}$ is a $K\left(\boldsymbol{p}^{\mathrm{rd}}\right)$ rational point. So $Y_{\boldsymbol{p}^{\mathrm{rd}}}$ is isomorphic to $\mathbb{P}_{z}^{1}$ over $K\left(\boldsymbol{p}^{\mathrm{rd}}\right)$.

Up to an affine change of variable over $K$, there is a copy of $\mathbb{P}^{1}=X$ over $K$ that parametrizes degree 7 Davenport pairs (over any nontrivial extension $L / K$ ).

\section{5. $j$-line covers for polynomial $\mathrm{PGL}_{3}(\mathbb{Z} / 3)$ monodromy}

We start by listing the 3 -tuples $\left(g_{1}, g_{2}, g_{3}\right)$ for $X_{i}$ in Table (5.1) that represent an absolute Nielsen class representative by tacking $g_{\infty}=(1 \cdots 13)^{-1}$ on the end $[\mathbf{F r} 99, \S 8]$. The rubric proceeds just as in the degree 7 case.

5.1. Degree 13 Davenport branch cycles $\left(g_{1}, g_{2}, g_{3}, g_{\infty}\right)$. The following elements are involutions fixing the hyperplane corresponding to the difference set. Each fixes one of the 9 points off the hyperplane. Compute directly possibilities for $g_{1}, g_{2}, g_{3}$ since each is a conjugate from this list:

$$
\begin{array}{lll}
(78)(511)(612)(913) ; & (311)(713)(68)(912) ; & (312)(58)(79)(1113) ; \\
(513)(69)(1112)(38) ; & (56)(37)(811)(1213) ; & (67)(811)(512)(313) ; \\
(35)(712)(613)(89) ; & (36)(59)(711)(813) ; & (57)(611)(813)(39) .
\end{array}
$$

Here is the table for applying the action of $q_{1}, q_{2}, q_{3}$. 
$X_{1}:(67)(811)(512)(313),(23)(134)(68)(910), \quad(12)(135)(612)(911)$ $X_{2}:(67)(811)(512)(313),(12)(135)(612)(911),(13)(54)(128)(1110)$ $X_{3}:(35)(712)(613)(89), \quad(16)(23)(137)(1210),(810)(1211)(62)(54)$ $X_{4}:(35)(712)(613)(89), \quad(810)(1211)(62)(54),(12)(63)(137)(118)$ $X_{5}$ :(56)(37)(911)(12 13), (13)(45)(812)(11 10), (23)(7 4)(1 8)(129) $X_{6}:(56)(37)(911)(1213), \quad(912)(23)(74)(18), \quad(82)(75)(19)(1110)$ $X_{7}$ :(56)(3 7)(911)(12 13), (19)(28)(75)(1011), (45)(3 8)(92)(121) $X_{8}:(56)(37)(911)(1213),(45)(38)(92)(121), \quad(122)(93)(74)(1110)$ $X_{9}:(89)(712)(136)(35), \quad(12)(63)(137)(118), \quad(13)(54)(128)(1110)$ $X_{10}$ :(6 7)(811)(512)(313), (13)(45)(12 8)(11 10), (6 8)(109)(413)(32) $X_{11}$ :(73)(56)(12 13)(911), (12)(75)(312)(89), (13)(54)(128)(1110) $X_{12}$ :(56)(3 7)(911)(12 13), (1011)(212)(39)(74), (12)(312)(75)(98) $X_{13}:(89)(613)(712)(35), \quad(13)(54)(128)(1110), \quad(23)(16)(713)(1012)$.

Here are the $j$-line branch cycle descriptions. From action (3.3) on Table (5.1)

$$
\begin{aligned}
\gamma_{0}^{*}=q_{1} q_{2} & =(153)(6913)(2811)(4710) \\
\gamma_{1}^{*}=q_{1} q_{2} q_{3} & =(14)(25)(36)(79)(810)(1112), \\
\gamma_{\infty}^{*}=q_{2} & =(1102)(31394)(51112876) .
\end{aligned}
$$

Again, you figure $\gamma_{1}^{*}$ from the product one condition. In analogy to Prop. 4.1 we prove properties (3.4) for degree 13 Davenport pairs. Denote $\left\langle\gamma_{0}^{*}, \gamma_{1}^{*}, \gamma_{\infty}^{*}\right\rangle$ by $G$.

Proposition 5.1. Then, $G=A_{13}$. With $\mathbf{C}^{*}=\left(\mathrm{C}_{0}, \mathrm{C}_{1}, \mathrm{C}_{\infty}\right)$ the conjugacy classes, respectively, of $\boldsymbol{\gamma}^{*}, \operatorname{Ni}\left(A_{13}, \mathbf{C}^{*}\right)^{\prime}$ (absolute equivalence classes with entries in order in $\mathbf{C}^{*}$ ) has one element. So, there is a unique cover $\bar{\psi}_{13}: X=X_{13} \rightarrow \mathbb{P}_{j}^{1}$ representing it (ramified over $\{0,1, \infty\}$ ). Restrict over $U_{\infty}$ for $\psi_{13}: X^{\infty} \rightarrow U_{\infty}$ equivalent to each $\mathcal{H}_{j} \rightarrow U_{\infty}, j=0,1,2,3$.

The projective curve $X$ has genus 0 and is not a modular curve. The spaces $\mathcal{H}_{j}$ are b-fine (not fine) moduli spaces over $K_{13}$. Their corresponding Hurwitz spaces are fine moduli spaces with a dense set of $K_{13}$ points. As a cover, however, $\bar{\psi}_{13}$ has definition field $\mathbb{Q}$, and $X$ has a dense set of $\mathbb{Q}$ points.

5.2. Proof of Prop. 5.1. First compute the genus $g_{13}$ of the curve in the cover presented by $\bar{\psi}_{13}$ to be 0 , from

$$
2\left(13+g_{13}-1\right)=\operatorname{ind}\left(\gamma_{0}^{*}\right)+\operatorname{ind}\left(\gamma_{1}^{*}\right)+\operatorname{ind}\left(\gamma_{\infty}^{*}\right)=4 \cdot 2+6+(2+3+5)=24 .
$$

Now we show why the geometric monodromy of the cover $\bar{\psi}_{13}$ is $A_{13}$. There are nine primitive groups of degree 13 [Ca56, p. 165]. Three affine groups $\mathbb{Z} / p \times{ }^{s} U$; each $U \leq(\mathbb{Z} / 13)^{*}$, with $U=\{1\}$, or having order 2 or order 3 . Then, there are six other groups $S_{13}, A_{13}$ and $\mathrm{PGL}_{3}(\mathbb{Z} / 3), \mathrm{PSL}_{3}(\mathbb{Z} / 3)$ with each of the last two acting on points and hyperplanes. The generators $\gamma_{0}^{*}$ and $\gamma_{1}^{*}$ are in $A_{13}$. It would be cute if the monodromy group were $\mathrm{PGL}_{3}(\mathbb{Z} / 3)$ (the same as of the covers represented by its points). We see, however, $\left(\gamma_{\infty}^{*}\right)^{6}$ fixes way more than half the integers in $\{1, \ldots, 13\}$. This is contrary to the properties of $\operatorname{PSL}_{3}(\mathbb{Z} / 3), \mathrm{PGL}_{3}(\mathbb{Z} / 3)$.

Now consider $\operatorname{Ni}\left(A_{13}, \mathbf{C}^{*}\right)^{\prime}$. Like $n=7$, assume triples of form $\left(g_{0}, g_{1}, \gamma_{\infty}^{*}\right)$. To simplify, conjugate by an $h \in S_{13}$ to change $\gamma_{\infty}^{*}$ to $(1234)(5678910)(111213)$ (keep its name the same). Its centralizer is $U=\langle(1234),(5678910),(11121314)\rangle$. Take $F=\{1,5,11\}$ as representatives, in order, of the three orbits $O_{1}, O_{2}, O_{3}$ of $\gamma_{\infty}^{*}$ on $\{1, \ldots, 13\}$. Suppose $g_{i}$ fixes $x_{i} \in\{1 \ldots, 13\}, i=0,1$. If $x_{0}$ and $x_{1}$ are in 
different $O_{k} \mathrm{~s}$, conjugate by $U$, and use transitivity of $\left\langle g_{0}, g_{1}\right\rangle$, to assume $x_{0}$ is one element of $F$ and $x_{1}$ another. We show the following hold.

(5.3a) Neither $g_{0}$ nor $g_{1}$ fixes an element of $O_{3}$.

(5.3b) The fixed point of $g_{0}$ is in $O_{1}$ or $O_{2}$, and of $g_{1}$ in the other.

(5.3c) With no loss we may assume either $x_{0}=5$ and $x_{1}=1$, or $x_{0}=1$ and $x_{1}=5$, and the latter is not possible.

5.2.1. Proof of (5.3a). Suppose $x_{0} \in O_{3}$; with no loss conjugating by $U$ take it to be 11. Then, $g_{0}=(1213 y) \cdots$ and $g_{1}=(1113)(y 12) \cdots$ (using product-one in the form $\left.\gamma_{\infty}^{*} g_{0} g_{1}=1\right)$. Then, however, $g_{0} g_{1}$ fixes $y$ : a contradiction, since $\gamma_{\infty}^{*}$ fixes nothing. Now suppose $x_{1} \in O_{3}\left(x_{1}=11\right)$. Then, $g_{0}=(1211 y) \cdots$ and $g_{1}=(y 13) \cdots$. Transitivity of $\left\langle\gamma_{\infty}^{*}, g_{0}\right\rangle$ prevents $y=13$. Conjugating by $U$ allows taking $y=1$ or $y=5$. If $y=1$, then you find $g_{0}=(12111)(2134) \cdots$ and $g_{1}=(113)(412)(23) \cdots$. Now, $\left\langle\gamma_{\infty}^{*}, g_{1}\right\rangle$ leaves $O_{1} \cup O_{3}$ stable, so is not transitive.

5.2.2. Proof of (5.3b), case $x_{0}, x_{1} \in O_{1}$. With no loss $x_{0}=1$ and $g_{1}=(14) \cdots$. This forces $g_{0}=(24 z) \cdots$, so under our hypothesis, (3) $g_{0}=3$. This forces $g_{1}=$ (14)(23) $\cdots$ and $\left\langle\gamma_{\infty}^{*}, g_{1}\right\rangle$ is stable on $O_{1}$.

5.2.3. Proof of (5.3b), case $x_{0}, x_{1} \in O_{2}$. With no loss

$$
x_{0}=5 \text { and } g_{1}=(610 z) \cdots, g_{1}=(105)(z 9) \cdots .
$$

We separately show $x_{1} \in\{6,7,8\}$ are impossible, and by symmetry, $x_{1} \in O_{2}$ is impossible. If $x_{1}=6$, then $z=7$, and $g_{1}=(6107)(89 w) \cdots, g_{1}=(105)(79)(w 8) \cdots$. The contradiction is that $g_{0} g_{1}$ fixes $w$.

If $x_{1}=7$, then $g_{0}=(610 z)(87 w) \cdots, g_{1}=(105)(z 9)(w 6) \cdots$. So neither $z$ nor $w$ can be 8 or 9: With no loss $w \in\{1,11\}$. If $w=11$, then with no loss $z \in\{12,1\}$. With $z=1$, (4) $g_{0} g_{1}=11$, a contradiction. If $z=12$,

$$
g_{0}=(61012)(8711)(139 u) \cdots, g_{1}=(105)(129)(116)(813) \cdots \text {. }
$$

Check: $u$ must be 13, a contradiction.

Finally, $w=1$ forces $g_{0}=(6102)(871)(39$ ? $) \cdots, g_{1}=(105)(29)(16)(84) \cdots$. So, $(9) g_{0} g_{1}=8$ forces $?=4$, and that forces $g_{1}$ to fix 3 , a contradiction to $x_{1}=7$.

That leaves $x_{1}=8$ and $g_{0}=(610 z)(98 w) \cdots, g_{1}=(105)(z 9)(w 7) \cdots$. Conjugate by $U$ to assume $w \in\{1,11\}$. The case $w=1$ forces

$$
g_{0}=(6104)(981)(273) \cdots, g_{1}=(105)(49)(17)(63) \cdots .
$$

Conclude: $\left\langle g_{0}, \gamma_{\infty}^{*}\right\rangle$ stabilizes $O_{1} \cup O_{2}$. If $w=11, g_{0}=(610 z)(9811) \cdots, g_{1}=$ $(105)(z 9)(117) \cdots$. In turn, this forces $z=10$ and 10 appears twice in $g_{1}$.

5.2.4. Proof of (5.3c). From (5.3a) and (5.3b), conjugate by $U$ for the first part of (5.3c). We must show $x_{0}=1, x_{1}=5$ is false. If this does hold, then

$$
g_{0}=(65 y)(24 z)(3 w ?) \cdots, g_{1}=(14)(y 10)(2 w)(3 z) \cdots .
$$

Note that $y \neq 2$, and 2 and 3 appear in distinct cycles in $g_{1}$ using $\left\langle\gamma_{\infty}^{*}, g_{1}\right\rangle$ is transitive. Here is the approach for the rest: Try each case where $y, z, w$ are in $O_{3}$. Whichever of $\{y, z, w\}$ we try, with no loss take this to be 11 .

Suppose $y=11$. Then, $g_{1} \gamma_{\infty}^{*}$ fixes 11 , contrary to $g_{0}$ not fixing it. Now suppose $z=11$. This forces $w=13$ and $\gamma_{\infty}^{*} g_{0}$ fixes 12 , though $g_{1}$ does not. Finally, suppose $w=11$, and get an analogous contradiction to that for $y=11$. With $y, z, w \in O_{2}$ we have $(3 w 11)$ a 3 -cycle of $g_{0}$. This forces $z=13$, a contradiction. 
5.2.5. Listing the cases with $x_{0}=5, x_{1}=1$. With these hypotheses:

$$
g_{0}=(21 y)(610 w) \cdots, g_{1}=(105)(y 4)(w 9) \cdots .
$$

We check that $y, w \notin O_{1}: y=3$ (or $w=3$ ) to get simple contradictions. Example: $w=3$ forces $(62)$ in $g_{1}$; forcing $y=7$, and $\left\langle g_{0}, \gamma_{\infty}^{*}\right\rangle$ is not transitive. Now check that $y \notin O_{3}$, but $w \in O_{3}$. Our normalization for being in $O_{3}$ allows $y=11$ :

$$
g_{0}=(2111)(610 w)(124 u)(313 v), g_{1}=(105)(114)(w 9)(213) \cdots .
$$

So, $w$ is 7 or 8 . The first forces $\gamma_{\infty}^{*} g_{0}$ to fix 6 , the second forces $g_{1} \gamma_{\infty}$ to fix 9 .

Now consider $y, w \in O_{2}$. If $y=7$ and $w=8$, then $g_{1} \gamma_{\infty}^{*}$ fixes 9 . If $y=8$ and $w=7$, then $\gamma_{\infty}^{*} g_{0}$ fixes 6 . We're almost done: Try $y \in O_{2}$, and $w=11$. Then $y=7$ or 8 . Try $y=7: g_{0}=(217)(61011)(3 z ?) \cdots, g_{1}=(105)(74)(119)(z 2) \cdots$. This forces $z=6$, putting $z$ in $g_{0}$ twice. So, $y=8$ : You find that from this start,

$$
g_{0}=(218)(61011)(1294)(1337), g_{1}=(105)(84)(119)(312)(27)(613)
$$

is forced, concluding that there is one element in $\mathrm{Ni}\left(A_{13}, \mathbf{C}^{*}\right)^{\prime}$.

It is easy that $A_{13}$ has no $\mathrm{PSL}_{2}(\mathbb{Z})$ quotient. The b-fine moduli is from $\mathcal{Q}^{\prime \prime}$ acting faithfully on the location of the 13-cycle conjugacy class (as with $n=7$ ). That $X^{\infty}$ does not have fine moduli follows from $\gamma_{0}^{*}$ and $\gamma_{1}^{*}$ having fixed points.

\section{Projective systems of Nielsen classes}

Let $F_{2}=\left\langle x_{1}, x_{2}\right\rangle$ be the free group on two generators. Consider two simple cases for a group $H$ acting faithfully on $F_{2}, H=\mathbb{Z} / 2(n=2)$ and $H=\mathbb{Z} / 3(n=3)$.

(6.1a) The generator of $H_{2}$ acts as $x_{i} \mapsto x_{i}^{-1}, i=1,2$.

(6.1b) The generator $1 \in \mathbb{Z} / 3=H_{3}$ acts as $x_{1} \mapsto x_{2}^{-1}$ and $x_{2} \mapsto x_{1} x_{2}^{-1}$.

We explain why these cases contrast extremely in achievable Nielsen classes. Let $\mathbf{C}_{2^{4}}$ be four repetitions of the nontrivial conjugacy class of $H_{2}$. Similarly, $\mathbf{C}_{ \pm 3^{2}}$ is two repetitions of each nontrivial $H_{3}$ class. Refer to $\mathbf{C}$ as $p^{\prime}$ conjugacy classes if a prime $p$ divides the orders of no elements in $\mathbf{C}$. Example: $\mathbf{C}_{ \pm 3^{2}}$ are $2^{\prime}$ classes.

6.1. Projective sequences of Nielsen classes. Assume $G^{*} \rightarrow G$ is a group cover, with kernel a $p$ group. Then $p^{\prime}$ conjugacy classes lift uniquely to $G^{*}[\mathbf{F r i 9 5 b}$, Part III]. This allows viewing $\mathbf{C}$ as conjugacy classes in appropriate covering groups.

Let $P$ be any set of primes. Denote the collection of finite $p$ group quotients of $F_{2}$, with $p \notin P$, by $\mathrm{Q}^{F_{2}}(P)$. Denote those stable under $H$ by $\mathrm{Q}^{F_{2}}(P, H)$. Consider inner Nielsen classes with some fixed $\mathbf{C}, P$ containing all primes dividing orders of elements in $\mathbf{C}$, and groups running over a collection from $\mathrm{Q}^{F_{2}}(P, H)$ :

$$
\mathcal{N}_{H}=\left\{\mathrm{Ni}(G, \mathbf{C})^{\text {in }}\right\}_{\left\{G=U \times{ }^{s} H \mid U \in \mathrm{Q}^{F_{2}}(P, H)\right\}} .
$$

Only $P=P_{n}=\{n\}$ for $n=2,3$ appear below, though we state a general problem.

Suppose for some $p, \mathcal{G}_{p, I}=\left\{U_{i}\right\}_{i \in I}$ is a projective subsequence of (distinct) $p$ groups from $\mathrm{Q}^{F_{2}}(P, H)$. Form a limit group $G_{p, I}=\lim _{\infty \leftarrow i} U_{i} \times{ }^{s} H$. Assume further, all Nielsen classes $\mathrm{Ni}\left(U_{i} \times{ }^{s} H, \mathbf{C}\right)$ are nonempty. Then, $\left\{\mathrm{Ni}\left(U_{i} \times{ }^{s} H, \mathbf{C}\right)^{\mathrm{in}}\right\}_{i \in I}$ forms a project system with a nonempty limit $\mathrm{Ni}\left(G_{p, I}, \mathbf{C}\right)$. If $\mathbf{C}$ has $r$ entries, then the Hurwitz monodromy group $H_{r}=\left\langle q_{1}, \ldots, q_{r-1}\right\rangle$ naturally acts (by (3.3)) on any of these inner (or absolute) Nielsen classes. We use just $r=4$.

Problem 6.1. Assume $I$ is infinite. What are the maximal groups $G_{p, I}$ from which we get nonempty limit Nielsen classes $\mathrm{Ni}\left(G_{p, I}, \mathbf{C}\right)$ ? 
We call such maximal groups $\mathbf{C} p$-Nielsen class limits. Prob. 6.7 refines this.

For $r=4$, consider maximal limits of projective systems of reduced components that have genus 0 or 1 . Allow $|I|$ here to be bounded. The strong Conjecture on Modular Towers $[\mathbf{F r S 0 4}, \S 1.2]$ specializes to $n=3$ to say this. Each such sequence should be bounded and there should be only finitely many (running over all $p \notin P_{3}$ ). Such genus 0 or 1 components have application. Ex. 6.2 is one such.

Any profinite pro- $p$ group $\hat{P}^{\prime}$ has a universal subgroup generated by $p$ th powers and commutators from $\hat{P}^{\prime}$. This is the Frattini subgroup, denoted $\Phi\left(P^{\prime}\right)$. The $k$ th iterate of this group is $\Phi^{k}\left(P^{\prime}\right)$. $\S 1.2 .2$ referenced reduced spaces $\left\{\overline{\mathcal{H}}_{k, p}^{\text {rd }}\right\}_{k \geq 0}$. These are the spaces for the Nielsen classes $\operatorname{Ni}\left(\hat{F}_{2, p} / \Phi^{k}\left(\hat{F}_{2, p}\right) \times{ }^{s} H_{3}, \mathbf{C}_{ \pm 3}\right)^{\text {in,rd }}$.

EXAmPle 6.2. Let $\varphi_{1}: G_{1}\left(A_{5}\right) \rightarrow A_{5}$ be the universal exponent 2 extension of $A_{5}$. We explain: If $\varphi: G^{*} \rightarrow A_{5}$ is a cover with abelian exponent 2 group as kernel, then there is a map $\psi: G_{1}\left(A_{5}\right) \rightarrow G^{*}$ with $\varphi \circ \psi=\varphi_{1}$. The space $\mathcal{H}_{2,1}^{\text {rd }}$ has six components [BFr02, Ex. 9.1, Ex. 9.3]. Two have genus 0, and two have genus 1. Let $K$ be a real number field. Then, there is only one possibility for infinitely many (reduced equivalence classes of) $K$ regular realizations of $G_{1}\left(A_{5}\right)$ with four branch points. It is that the genus 1 components of $\overline{\mathcal{H}}_{2,1}^{\text {rd }}$ have definition field $K$ and infinitely many $K$ rational points. The genus 0 components here have no real points. [FrS04] explains this in more detail.

6.2. $\mathcal{N}_{2} \stackrel{\text { def }}{=}\left\{\mathrm{Ni}\left(G, \mathbf{C}_{2^{4}}\right)^{\text {in }}\right\}_{G \in \mathbf{Q}^{F_{2}(2) \times{ }^{s} H_{2}}}$. The first sentence of Prop. 6.3 restates an argument from [Fri95b, $\S 1$.A].

6.2.1. Achievable Nielsen classes from modular curves. Let $\boldsymbol{z}=\left\{z_{1}, \ldots, z_{4}\right\}$ be any four distinct points of $\mathbb{P}_{z}^{1}$, without concern to order. As in $\S 2.2 .1$ choose a set of (four) classical generators for the fundamental group of $\mathbb{P}_{z}^{1} \backslash \boldsymbol{z}=U_{\boldsymbol{z}}$.

This group identifies with the free group on four generators $\boldsymbol{\sigma}=\left(\sigma_{1}, \ldots, \sigma_{4}\right)$, modulo the product-one relation $\sigma_{1} \sigma_{2} \sigma_{3} \sigma_{4}=1$. Denote its completion with respect to all normal subgroups by $\hat{F}_{\boldsymbol{\sigma}}$. Let $\hat{\mathbb{Z}}_{p}$ (resp. $\hat{F}_{2, p}$ ) be the similar completion of $\mathbb{Z}$ (resp. $F_{2}$ ) by all normal subgroups with $p$ group quotient.

Proposition 6.3. Let $\hat{D}_{\boldsymbol{\sigma}}$ be the quotient of $\hat{F}_{\boldsymbol{\sigma}}$ by the relations

$$
\sigma_{i}^{2}=1, i=1,2,3,4\left(\text { so } \sigma_{1} \sigma_{2}=\sigma_{4} \sigma_{3}\right) \text {. }
$$

Then, $\prod_{p \neq 2} \hat{\mathbb{Z}}_{p}^{2} \times{ }^{s} H_{2} \equiv \hat{D}_{\boldsymbol{\sigma}}$. Also, $\hat{\mathbb{Z}}_{p}^{2} \times{ }^{s} H_{2}$ is the unique $\mathbf{C}_{2^{4}}$ p-Nielsen class limit.

Proof. We show combinatorially that $\hat{D}_{\boldsymbol{\sigma}}$ is $\hat{\mathbb{Z}}^{2} \times{ }^{s} H_{2}$ and that $\sigma_{1} \sigma_{2}$ and $\sigma_{1} \sigma_{3}$ are independent generators of $\hat{\mathbb{Z}}^{2}$. Then, $\sigma_{1}$ is a generator of $H_{2}$ which we regard as $\{ \pm 1\}$ acting on $\hat{\mathbb{Z}}^{2}$ by multiplication. First: $\sigma_{1}\left(\sigma_{1} \sigma_{2}\right) \sigma_{1}=\sigma_{2} \sigma_{1}$ shows $\sigma_{1}$ conjugates $\sigma_{1} \sigma_{2}$ to its inverse. Also,

$$
\left(\sigma_{1} \sigma_{2}\right)\left(\sigma_{1} \sigma_{3}\right)=\left(\sigma_{1} \sigma_{3}\right) \sigma_{3}\left(\sigma_{2} \sigma_{1}\right) \sigma_{3}=\left(\sigma_{1} \sigma_{3}\right)\left(\sigma_{1} \sigma_{2}\right)
$$

shows the said generators commute. The maximal pro- $p$ quotient is $\mathbb{Z}_{p}^{2} \times{ }^{s}\{ \pm 1\}$.

We have only to show Nielsen classes with $G=U \times{ }^{s} H_{2}$, and $U$ an abelian quotient of $\mathbb{Z}^{2}$, are nonempty. It suffices to deal with the cofinal family of $U \mathrm{~s}$, $\left(\mathbb{Z} / p^{k+1}\right)^{2}, p \neq 2$. $\S 6.2 .2$ has two proofs.

Remark 6.4. Any line (pro-cyclic group) in $\mathbb{Z}_{p}^{2}$ produces a dihedral group $D_{p^{\infty}}$ from multiplication by -1 on this line. 
6.2.2. Nonempty Nielsen classes in Prop. 6.3. In $G_{p^{k+1}}=\left(\mathbb{Z} / p^{k+1}\right)^{2} \times{ }^{s}\{ \pm 1\}$, $\left\{(-1 ; \boldsymbol{v}) \mid \boldsymbol{v} \in\left(\mathbb{Z} / p^{k+1}\right)^{2}\right\}$ are the involutions. Write $\boldsymbol{v}=(a, b), a, b \in \mathbb{Z} / p^{k+1}$. The multiplication $\left(-1 ; \boldsymbol{v}_{1}\right)\left(-1 ; \boldsymbol{v}_{2}\right)$ yields $\left(1 ; \boldsymbol{v}_{1}-\boldsymbol{v}_{2}\right)$ as in the matrix product

$$
\left(\begin{array}{cc}
-1 & \boldsymbol{v}_{1} \\
0 & 1
\end{array}\right)\left(\begin{array}{cc}
-1 & \boldsymbol{v}_{2} \\
0 & 1
\end{array}\right)
$$

We have an explicit description of the Nielsen classes $\mathrm{Ni}\left(G_{p^{k+1}}, \mathbf{C}_{2^{4}}\right)$. Elements are 4 -tuples $\left(\left(-1 ; \boldsymbol{v}_{1}\right), \ldots,\left(-1 ; \boldsymbol{v}_{4}\right)\right)$ satisfying two conditions from $\S 2.2$ :

(6.2a) Product-one: $\boldsymbol{v}_{1}-\boldsymbol{v}_{2}+\boldsymbol{v}_{3}-\boldsymbol{v}_{4}$; and

(6.2b) Generation: $\left\langle\boldsymbol{v}_{i}-\boldsymbol{v}_{j}, 1 \leq i, j \leq 4\right\rangle=\left(\mathbb{Z} / p^{k+1}\right)^{2}$.

Apply conjugation in $G_{p^{k+1}}$ to assume $\boldsymbol{v}_{1}=0$. Now take $\boldsymbol{v}_{2}=(1,0), \boldsymbol{v}_{3}=(0,1)$ and solve for $\boldsymbol{v}_{4}$ from the product-one. This shows the Nielsen class is nonempty. To simplify our discussion we have taken inner Nielsen classes. What really makes an interesting story is the relation between inner and absolute Nielsen classes. Use the natural inclusion $G_{p^{k+1}} \triangleleft\left(\mathbb{Z} / p^{k+1}\right)^{2} \times{ }^{s} \mathrm{GL}_{2}\left(\mathbb{Z} / p^{k+1}\right)$ regarding both groups as permutations of $\left(\mathbb{Z} / p^{k+1}\right)^{2}$.

A general theorem in [FV91] applies here. It says the natural map from $\mathcal{H}\left(G_{p^{k+1}}, \mathbf{C}_{2^{4}}\right)^{\text {in }} \rightarrow \mathcal{H}\left(G_{p^{k+1}}, \mathbf{C}_{2^{4}}\right)^{\text {abs }}$ is Galois with group $\mathrm{GL}_{2}\left(\mathbb{Z} / p^{k+1}\right) /\{ \pm 1\}$. We give our second proof for nonempty Nielsen classes to clarify the application of [Se68, IV-20]. This shows, depending on the $j$-value of the 4 branch points for the cover $\varphi_{p}: X_{p} \rightarrow \mathbb{P}_{z}^{1}$, we can say explicit things about the fiber of $\mathcal{H}\left(G_{p^{k+1}}, \mathbf{C}_{2^{4}}\right)^{\text {in }} \rightarrow \mathcal{H}\left(G_{p^{k+1}}, \mathbf{C}_{2^{4}}\right)^{\text {abs }}$ over $\boldsymbol{p} \in \mathcal{H}\left(G_{p^{k+1}}, \mathbf{C}_{2^{4}}\right)^{\text {abs }}$.

We will now display the cover $\varphi_{\boldsymbol{p}}$. Let $E$ be any elliptic curve in Weierstrass normal form, and $\left[p^{k+1}\right]: E \rightarrow E$ multiplication by $p^{k+1}$. Mod out by the action of $\{ \pm 1\}$ on both sides of this isogeny to get

$$
E /\{ \pm 1\}=\mathbb{P}_{w}^{1} \stackrel{\varphi_{p^{k+1}}}{\longrightarrow} E /\{ \pm 1\}=\mathbb{P}_{z}^{1},
$$

a degree $p^{2(k+1)}$ rational function. Compose $E \rightarrow E /\{ \pm 1\}$ and $\varphi_{p^{k+1}}$ for the Galois closure of $\varphi_{p^{k+1}}$. This geometrically shows $\mathrm{Ni}\left(G_{p^{k+1}}, \mathbf{C}_{2^{4}}\right) \neq \emptyset$. If $E$ has definition field $K$, so does $\varphi_{p^{k+1}}$. We may, however, expect the Galois closure field of $\varphi_{p^{k+1}}$ to have interesting constants, from the definition fields of $p^{k+1}$ division points on $E$. This is the subject of Serre's Theorem and [Fr04, §5.2-5.3].

6.3. $\mathcal{N}_{3} \stackrel{\text { def }}{=}\left\{\mathrm{Ni}\left(G, \mathbf{C}_{ \pm 3^{2}}\right)^{\mathrm{in}}\right\}_{G \in \mathbf{Q}^{F_{2}}(3) \times{ }^{s} H_{3}}$. Prop. 6.5 says, unlike $n=2$, for any $G \in \mathcal{N}_{3}, \operatorname{Ni}\left(G, \mathbf{C}_{ \pm 3^{2}}\right)^{\text {in }}$ is nonempty. This vastly differs from the conclusion of Prop. 6.3. Our proof combines Harbater-Mumford reps. (Ex. 6.6) with the cyclic action of $H_{3}$ on $(\mathbb{Z} / p)^{2}$. We make essential use of the Frattini property.

Proposition 6.5. $\hat{F}_{2, p} \times{ }^{s} H_{3}$ is the unique $\mathbf{C}_{ \pm 3^{2}}$ p-Nielsen class limit.

Proof. First we show Nielsen classes with $G=G_{p}=(\mathbb{Z} / p)^{2} \times{ }^{s} H_{3}(p \neq 2)$ are nonempty by showing each contains an $\mathrm{H}-\mathrm{M}$ rep. Let $\langle\alpha\rangle=\mathbb{Z} / 3$, with the notation on the left meaning multiplicative notation (so, 1 is the identity). The action of $\alpha$ is from (6.1b). For multiplication in $G_{p}$ use the analog of that in $\S 6.2 .2$.

Suppose $g_{1}=\left(\alpha, \boldsymbol{v}_{1}\right), g_{2}=\left(\alpha, \boldsymbol{v}_{2}\right) \in G$ generate $G$. Then, $\left(g_{1}, g_{1}^{-1}, g_{2}, g_{2}^{-1}\right)$ is in $\operatorname{Ni}\left(G, \mathbf{C}_{ \pm 3^{2}}\right)^{\text {in }}$. Further this element is an H-M rep. Conjugate this 4-tuple by $\left(1, \boldsymbol{v}_{3}\right)$ (for inner equivalence) to change $g_{1}$ to $\left(\alpha, \boldsymbol{v}_{1}+\boldsymbol{v}_{3}-\alpha\left(\boldsymbol{v}_{3}\right)\right)$. As $I_{2}-\alpha$ is invertible on $(\mathbb{Z} / p)^{2}$ we can choose $\boldsymbol{v}_{3}$ so $\boldsymbol{v}_{1}=\mathbf{0}$. To find such generators, consider $g_{1} g_{2}^{-1}=\left(1,-\boldsymbol{v}_{2}\right)$ and $g_{1}^{2} g_{2}=\left(1, \alpha^{-1}\left(v_{2}\right)\right)$. So, $g_{1}, g_{2}$ are generators precisely if 
$\left\langle-\boldsymbol{v}_{2}, \alpha^{-1}\left(v_{2}\right)\right\rangle=(\mathbb{Z} / p)^{2}$. Such a $\boldsymbol{v}_{2}$ exists because the eigenvalues of $\alpha$ are distinct. So $(\mathbb{Z} / p)^{2}$ is a cyclic $\langle\alpha\rangle$ module. If $x^{2}+x+1 \bmod p$ has no solution, then $\langle\alpha\rangle$ acts irreducibly and any $\boldsymbol{v}_{2} \neq \mathbf{0}$ works. From quadratic reciprocity this is equivalent to -3 is not a square $\bmod p$, or $p \equiv-1 \bmod 3$.

Now consider any Nielsen class in $\mathcal{N}_{3}$ defined by $G=U \times{ }^{s} H_{3}$ with $U$ having $(\mathbb{Z} / p)^{2}$ as a quotient. There is a surjective map $\psi: G \rightarrow(\mathbb{Z} / p)^{2} \times{ }^{s} H_{3}$, and it is a Frattini cover. So, if $g_{1}^{\prime}, g_{2}^{\prime}$ are generators of $(\mathbb{Z} / p)^{2} \times{ }^{s} H_{3}$ given by the proof above, then any respective order 3 lifts of $g_{1}^{\prime}, g_{2}^{\prime}$ to $g_{1}, g_{2} \in G$ will automatically generate $G$. Therefore the representative $\left(g_{1}, g_{1}^{-1}, g_{2}, g_{2}^{-1}\right)$ of the Nielsen class $\operatorname{Ni}\left(G, \mathbf{C}_{ \pm 3^{2}}\right)^{\text {in }}$ lifts $\left(g_{1}^{\prime},\left(g_{1}^{\prime}\right)^{-1}, g_{2}^{\prime},\left(g_{2}^{\prime}\right)^{-1}\right)$. This shows all Nielsen classes are nonempty.

6.4. Projective cusp types. Let $r=4$ and $\boldsymbol{g} \in \mathrm{Ni}(G, \mathbf{C})^{\mathrm{in}, \mathrm{rd}}$ (or any reduced equivalence). Then, the orbit of $\boldsymbol{g}$ under the cusp group $\mathrm{Cu}_{4} \stackrel{\text { def }}{=}\left\langle q_{2}, \mathcal{Q}^{\prime \prime}\right\rangle$ interprets as a cusp of the corresponding $j$-line cover. Certain Nielsen class representatives define especially useful cusps. We define these relative to a prime $p$. [FrS04, §5.2] calls these $\mathrm{g}-p^{\prime}$ cusps, and when $r=4$, their representatives $\boldsymbol{g}=\left(g_{1}, \ldots, g_{4}\right)$ in reduced Nielsen classes have the following property:

(6.3) Both $H_{2,3}(\boldsymbol{g}) \stackrel{\text { def }}{=}\left\langle g_{2}, g_{3}\right\rangle$ and $H_{1,4}(\boldsymbol{g})=\left\langle g_{1}, g_{4}\right\rangle$ are $p^{\prime}$ groups; their orders are prime to $p$.

Recall the shift $\mathbf{s h}=q_{1} \cdots q_{r-1}$ as an operator on Nielsen classes: For

$$
\boldsymbol{g} \in \mathrm{Ni}(G, \mathbf{C}),(\boldsymbol{g}) \mathbf{s h}=\left(g_{2}, \ldots, g_{r}, g_{1}\right) .
$$

EXAMPLE 6.6 (H-M reps.). Suppose $h_{1}$ and $h_{2}$ are elements generating a group $G$ and consider the 4 -tuple $\boldsymbol{h}=\left(h_{1}, h_{1}^{-1}, h_{2}, h_{2}^{-1}\right)$. If $\boldsymbol{h} \in \mathrm{Ni}(G, \mathbf{C})$, we say $\boldsymbol{h}$ is a Harbater-Mumford representative (H-M rep.) of the Nielsen class. If $\mathbf{C}$ are $p^{\prime}$ conjugacy classes, then $(\boldsymbol{h}) \mathbf{s h}$ is a a representative for a $p^{\prime}$ cusp.

[FrS04, Prop. 5.1] generalizes how we use H-M reps., in Prop. 6.5 as follows. Suppose level 0 of a Modular Tower, for the prime $p$, has representatives of $g-p^{\prime}$ cusps. Then applying Schur-Zassenhaus gives projective systems of $g-p^{\prime}$ cusps. This is the only method we know to show there are nonempty Nielsen classes at all levels.

So, we don't know whether g- $p^{\prime}$ cusps are necessary for a result like Prop. 6.5. For this case alone we pose a problem that aims at deciding that. Use the action of $H_{4}=\left\langle q_{1}, q_{2}, q_{3}\right\rangle$ in $\S 6.1$ (from (3.3)). For Nielsen classes from $\mathcal{N}_{3}$, it is easy to show all representatives of $\mathrm{g}-p^{\prime}$ cusps $(p \neq 3)$ must be shifts of H-M reps.

Problem 6.7. Suppose $O$ is an $H_{4}$ orbit on $\operatorname{Ni}\left(\hat{F}_{2, p} \times{ }^{s} H_{3}, \mathbf{C}_{ \pm 3}\right)^{\text {in,rd }}$. Must $O$ contain the shift of an H-M rep.?

\section{Relating Problem ${ }_{1}^{g=0}$ and Problem $_{2}^{g=0}$}

Our last topic discusses, using the work of others, what might be the meaning and significance of an intrinsic uniformizer for a 1-dimensional genus 0 moduli space.

7.1. Explicit equations. As $\S 2.2 .2$ notes, our computations were with pencil and paper. Many saw the then unpublished pieces we used from [Fr95a, p. 349] and $[\mathbf{F r} \mathbf{9 9}, \S 8]$ in 1969 at the writing of $[\mathbf{F r} \mathbf{7 3}]$. Precisely relating to the $j$-line is more recent, though you see these computations are easy. Further, they create a framework for finding a parameter for a moduli cover of the $j$-line having an internal interpretation. 
7.1.1. Couveignes calculations. Jean-Marc Couveignes used computer-assisted calculations to find equations for Davenport pairs [CoCa99]. He also gave a technique for uniformizing some moduli of the type we are considering. Necessarily, the moduli was of genus 0 covers, and the moduli space of genus 0 [Co00]. Prop. 4.1 and Prop. 5.1 produce a natural geometry behind the genus 0 moduli from Davenport's arithmetically defined problem. We compare our computation with that from [CoCa99], whose tool was PARI Ver. 1.920.24 with Maple used as a check.

Equations for those degree 7, 13 and 15 polynomials are in [CoCa99, §5.1], [CoCa99, §5.3] and [CoCa99, §5.4]. [Fr80, p. 593] has Birch's brute force calculation of degree 7 Davenport pairs. He and Guy also did the degree 11 case (three branch point covers, so up to reduced equivalence this is just one pair of polynomials). Degree 13 is the most interesting. There the difference set argument produces the nontrivial intertwining of four polynomials for each point of the space $X_{13}^{\infty}$. As the [CoCa99] calculations take considerable space, we don't repeat them. Still, a statistical comparison indicates the extra complexity (supported by theory) in the degree 13 case. Here is the character count for Couveignes' expression for the general polynomial in each case (not counting spaces):

(7.1) degree 7: 146 ; degree 13: 1346 ; degree 15: 819.

7.1.2. Significance of the $j$-line cover. Neither Birch nor Couveignes relates their equations to the $j$-line. Still, two points help compare [Co00] with our goals.

(7.2a) Using [GHP88], Harbater patching can sometimes produce the equation for the general member a family of genus 0 covers.

(7.2b) Using formal fibers of a moduli problem avoids direct computation of a possibly large base extension (when no version of rigidity holds).

I think (7.2a) refers to moduli of genus 0 covers, when a Nielsen class gives data to normalize a parameter for each cover in the family. We explain below this computational handle that starts from degenerate situations and at the cusps.

Grothendiecks' original method, reviewed in [Fr99, §3.6], is totally compatible. Except, applying Grothendieck's method requires a computationally inexact Artin approximation to achieve an algebraic deformation. It is around that last step that Couveignes uses his genus 0 assumptions.

The example of [CoCa99, p. 48] has some version of reduced parameters, though over the $\lambda$-line, not the $j$-line. [CoCa99, p. 56] gives the practical sense of (7.2b). We use his formal deformation parameter $\mu$. His example serves no exterior problem. Still, it illustrates the computational technique. Though we admire it, we want to show why there are many constraints on its use to compute equations.

7.1.3. Constraints in Couveignes' example. The group for his Nielsen classes is $S_{7}$, and his conjugacy classes $\mathbf{C}^{c}$ are those represented by the entries of

$$
\boldsymbol{g}_{\mu}=\left((234)(67),(1256),(17),(234567)^{-1}\right) .
$$

He interpolates between two 3 branch point covers with respective branch cycles:

$$
\begin{aligned}
& \boldsymbol{g}_{0}=\left((234)(67),(1256),(1234567)^{-1}\right) \text { and; } \\
& \boldsymbol{g}_{1}=\left((1234567),(17),(234567)^{-1}\right) .
\end{aligned}
$$

His four conjugacy classes are all distinct. Then, the reduced family of absolute covers in the Nielsen class, as a $j$-line cover, automatically factors through the $\lambda$ line as $\bar{\psi}^{c}: \overline{\mathcal{H}}\left(S_{7}, \mathbf{C}^{c}\right)^{\text {abs,rd }} \rightarrow \mathbb{P}_{\lambda}^{1}$. He finds explicit equations $\varphi_{0}$ and $\varphi_{1}$ for the unique covers branched over $\{0,1, \infty\} \in \mathbb{P}_{j}^{1}$ with branch cycles represented by $\boldsymbol{g}_{0}$ 
and $\boldsymbol{g}_{1}$. An analyst would view this as forming $\varphi_{\mu}: \mathbb{P}_{w_{\mu}}^{1} \rightarrow \mathbb{P}_{z}^{1}$ as a function of $\mu \in(0,1)$ so that as $\mu \mapsto 0$ (resp. 1) $\varphi_{\mu}$ degenerates to $\varphi_{0}$ (resp. $\left.\varphi_{1}\right)$.

Topologically this is a coalescing, respectively, of the first two (last two) branch points. Realize, however, compatible with the statement in $\S 2.2 .1$, this is not an algebraic process. We want equations for $\varphi_{\mu}$ in the coordinates of the parameter space $\overline{\mathcal{H}}\left(S_{7}, \mathbf{C}^{c}\right)$ abs,rd . [CoCa99, p. 43-48] refers to [DFr94, Thm. 4.5] to compute the action of the $\lambda$-line (not $j$-line) version of $\gamma_{0}^{*}, \gamma_{1}^{*}, \gamma_{\infty}^{*}$ (as in Propositions 4.1 and 5.1). He concludes $\overline{\mathcal{H}}\left(S_{7}, \mathbf{C}^{c}\right)^{\text {abs,rd }}$ has genus 0 . Dropping reduced equivalence gives a fine moduli space (reason as in $\S 4.2 .1$ ).

To track the $\lambda$-line, we must express coordinates for $\boldsymbol{p} \in \mathcal{H}\left(S_{7}, \mathbf{C}^{c}\right)^{\text {abs,rd }}$ in the algebraic closure of $\mathbb{Q}(\lambda)$. This space is irreducible, from the analog of $\gamma_{0}, \gamma_{1}, \gamma_{\infty}$ acting transitively. For the same reason as in our Davenport pair examples, reduced equivalence gives a b-fine moduli space $(\S 4.2)$. These are hypotheses that satisfy the easiest case of braid rigidity. So, the cardinality of the reduced Nielsen class equals the degree of those coordinates over $\mathbb{Q}(\lambda)$ ([Fr77, Cor. 5.3], [MM99, Thm. 5.3] or [Vö96, §10.3.1]; responding to the potential problem of $(7.2 \mathrm{~b}))$.

[Co00, p. 50] uses a set of classical generators (as in §2.2.1) around branch points. Then, the effect of coalescing a pair of branch points, say, as $\mu \mapsto 1$ interprets simply. It is as if you replaced the $3 \mathrm{rd}$ and 4 th of the classical generators by their product. Still, this is topological, not algebraic, data.

For that he normalizes a parameter $w_{\mu}$ (to appear in $\varphi_{\mu}\left(w_{\mu}\right)$ ) by selecting 3 distinguished points on the cover $\varphi_{\mu}$. These correspond to selecting 3 distinguished disjoint cycles in the branch cycles $\boldsymbol{g}_{\mu}$ in (7.3). He wants $w_{\mu}=w_{\mu, 1}$ to survive (in the limit $\mu \mapsto 1$ ) to give $w_{1}$ (similarly with $w_{0}$ ). So, the points he chooses must survive the coalescing. It is a constraint on the explicit equations that he can pick three such points. It is a separate constraint that he can do the same on the other limit $\mu \mapsto 0$, producing a parameter $w_{\mu, 0}$ for $\mu$ near 0 .

Here in [Co00, p. 50-51] a reader might have difficulty (see the reference). Naming the three points (labeled $V_{3}, V_{2}, V_{6}$ ), where $w_{\mu, 1}$ takes respective values $0,1, \infty$ (chosen for $\mu \mapsto 1$ ) appears after their first use in equations. That is because of a misordering of the printed pages. He analytically continues these choices along $\mu \in(0,1)$. They assure $w_{\mu, 0} / w_{\mu, 1}$ is $w_{\mu, 0}\left(V_{2}\right) / w_{\mu, 1}\left(V_{2}\right)$, expressed in meaningful constants from ramification of $\bar{\psi}^{c}$, as a function of $\mu$. [Co00] then explains what to do with expressions for $\varphi_{\mu}\left(w_{\mu, k}\right), k=0,1$ expressed as local power series. He uses their truncation up to the necessary degree of accuracy for their determination from the algebraic conditions. Details on the genus 0 moduli space come into play to precisely express coefficients of a general member of the family.

7.1.4. Using geometric compactifications. Couveignes applied coordinates from [GHP88] for his explicit compactification. Many use a compactification around cusps, placing over the cusp something called an admissible cover of (singular) curves. Arithmetic applications require knowing that the constant field's absolute Galois group detects the situation's geometry. My version is a specialization sequence [Fri95b, Thm. 3.21]. This gives meaningful action on projective sequences of cusps. The goal is to see exactly how $G_{\mathbb{Q}}$ acts from its preserving geometric collections like $g-p^{\prime}$ cusps $(\S 6.1)$.

My treatment, however, did not aim at explicit equations. [DDE04, Thm. 1.3 and Thm. 4.1] gave a treatment of [Fri95b, Thm. 3.21] using admissible covers (the version in $[\mathbf{W e} 99])$. For that reason, they compactify with a family that has $H-M$ 
admissible covers around the H-M cusps. (They use Hurwitz, not reduced Hurwitz, spaces.) A corollary of [Fri95b, Thm. 3.21] has simple testable hypotheses that guarantee there is a unique component of the moduli space containing $\mathrm{H}-\mathrm{M}$ cusps (so it is over $\mathbb{Q}$ ). Those hypotheses rarely hold if $r=4$.

So, I wish someone could do the following.

Problem 7.1. Approach the genus 1 components in Ex. 6.2 as did Couveignes for his examples.

Couveignes' explicit constrains fail miserably here. Ex. 6.2 is a family of very high genus curves with no distinguished disjoint cycles in their branch cycle descriptions. Still, the topics of [DDE04] and [We99] are relevant. The two genus 1 components of Ex. 6.2 are both H-M rep. components. The two components come from corresponding orbits for $H_{4}$ acting on inner Nielsen class orbits. They are, however, not total mysteries: An outer automorphism of $G_{1}\left(A_{5}\right)$ joins the orbits. (None of that is obvious; $[\mathbf{F r S 0 4}, \S 7]$ will have complete documentation.)

7.1.5. An intrinsic uniformizing parameter. Interest in variables separated polynomials $f(x)-g(y)$, those $\S 2.1$ calls Davenport pairs, first came from factorization questions ([DLS61] and [DS64]). As [Fr04, §1.1.2] explains, Davenport's own questions showed his interest in the finite field properties. That exhibited them with delicate arithmetic properties. Given that, we should express a uniformizing parameter, for $X_{n}(n=7,13,15)$ using the arithmetic behind their investigation. We don't know if there is such, though [Fr04] suggests some based on functions in $(x, y)$ (satisfying $f(x)-g(y)=0$ ) that are constant along fibers to the space $X_{n}$.

7.2. A piece of John Thompson's influence. I speak only of John's influence in specific mathematical situations related to this paper. John and I had a conversation in 1986 on the way to lunch at University of Florida. This one conversation brings a luminous memory, so singular it may compress many conversations.

7.2.1. John's formation of Problem ${ }_{2}^{g=0}$. As we walked, I summarized the group theory from solving several problems, like Davenport's. Each came with an an equation that we could rewrite as a phrase on primitive (genus 0) covers. My conclusion: There were always but finitely many rational function degree counterexamples to the most optimistic hopes. Yet, there were some counterexamples.

Further, to solve these problems required nonobvious aspects of groups. For example: In Davenport's problem, we needed difference sets and knowledge of all the finite groups with two distinct doubly transitive permutation representations that were equivalent as group representations (I got this from [CKS76]). Quite like the classification, many simple groups and some new number theory, impinged on locating the polynomial Davenport pairs. That was my pitch.

John responded that he was seized with the underlying problem. His initial formulation was this. Suppose $G$ is a composition factor of the covering monodromy of $\varphi: X \rightarrow \mathbb{P}_{z}^{1}$ with $X$ of genus $g$. Then, it is a genus $g$ group.

Problem 7.2. We fix an integer $g$ and exclude alternating and cyclic groups. Show: Only finitely many simple groups have genus $g$.

As above, the genus 0 primitive cover case suffices. This was my encouragement for the project. It is nontrivial to grab a significant monodromy group at random. (You will always get the excluded groups.) Still, the genus 0 problem would display exceptions. These should contribute conspicuously, as happened with the Schur, 
Davenport and HIT problems. That is, a general theorem would have sporadic counterexamples. While they might be baffling, they would nevertheless add to the perceived depth of the result. Especially they would guide situations of higher genus, and in positive characteristic. Example: I suggested there would be new primitive rational functions beyond those coming from elliptic curves, that had the Schur cover property: Giving one-one maps on $\mathbb{P}_{z}^{1}\left(\mathbb{F}_{p}\right)$ for $\infty$-ly many primes $p$.

John suggested we work toward this immediately. I had hoped, then, he would be interested in my approach to using the universal $p$-Frattini cover of a finite group. My response was that Bob Guralnick enjoyed this type of problem and knew immensely more about the classification than I. So was born Problem ${ }_{2}^{g=0}$, and the collaboration of Guralnick-Thompson.

7.2.2. Progress on Problem $m_{2}^{g=0}$. John showed me the initial list from his work with Bob on the affine group case. The display mode was groups presented by branch cycle generators: an absolute Nielsen class $(\$ 2.2)$. I noted three degree 25 rational functions requiring just the branch cycle argument [Fr77, p. 62] to see they had the Schur cover property. (You measure this by distinguishing between its arithmetic and geometric monodromy groups.) meant it did not come from elliptic curves, or twists of cyclic or Chebychev polynomials.

We now have a list of Nielsen classes sporadic for the Schur property (Schursporadic; [GMS03, Thm. 1.4]). [Fr04, §7.2.1] uses this as we did Müller's polynomial 0 -sporadic list in this paper.

Problem $_{2}^{g=0}$ in John's form is true. There are only finitely many sporadic genus 0 groups. Most major contributors are in this chronological list: [GT90], [As90], [LS91], [S91], [GN92], [GN95], [LSh99], and [FMa01]. Reverting to the primitive case parceled the task through the 5-branch Aschbacher-O'Nan-Scott classification of primitive groups [AS85].

7.2.3. Guralnick's optimistic conjecture. Yet, there is an obvious gap between the early papers and the two at the end. The title of [LSh99] reveals it did not list examples precisely as John did at the beginning. We can't yet expect the exceptional Chevalley groups to fall easily to such explicitness; you can't grab your favorite permutation representation with them. Still, composition factors are one thing, actual genus 0 primitive monodromy groups another.

Definition 7.3. We say $T: G \rightarrow S_{n}$, a faithful permutation representation, with properties (7.5) and (7.6) is 0-sporadic.

Denote $S_{n}$ on unordered $k$ sets of $\{1, \ldots, n\}$ by $T_{n, k}: S_{n} \rightarrow S_{\left(\begin{array}{c}n \\ k\end{array}\right)}$ by $\left(T_{n, 1}\right.$ the standard action). Alluding to $S_{n}$ (or $A_{n}$ ) with $T_{n, k}$ nearby refers to this presentation. In (7.6), $V_{a}=(\mathbb{Z} / p)^{a}$ ( $p$ a prime). Use $\S 6.2 .2$ for semidirect product in the $T_{V_{a}}$ case on points of $V_{a} ; C$ can be $S_{3}$. For the second $\left(A_{n}, T_{n, 1}\right)$ case, $T: G \rightarrow S_{n^{2}}$.

(7.5) $(G, T)$ is the monodromy group of a primitive $(\S 2.2)$ compact Riemann surface cover $\varphi: X \rightarrow \mathbb{P}_{z}^{1}$ with $X$ of genus 0 .

(7.6) $(G, T)$ is not in this list of group-permutation types.

- $\left(A_{n}, T_{n, 1}\right): A_{n} \leq G \leq S_{n}$, or $A_{n} \times A_{n} \times^{s} \mathbb{Z} / 2 \leq G \leq S_{n} \times S_{n} \times{ }^{s} \mathbb{Z} / 2$.

- $\left(A_{n}, T_{n, 2}\right): A_{n} \leq G \leq S_{n}$.

- $T_{V_{a}}: G=V \times{ }^{s} C, a \in\{1,2\},|C|=d \in\{1,2,3,4,6\}$ and $a=2$ only if $d$ does not divide $p-1$.

Rational functions $f \in \mathbb{C}(x)$ represent 0 -sporadic groups by $f: \mathbb{P}_{x}^{1} \rightarrow \mathbb{P}_{z}^{1}$. We say $(G, T)$ is polynomial 0-sporadic, if some $f \in \mathbb{C}[x]$ represents it. 
Definition 7.4. Similarly, we say $(G, T)$ is $g$-sporadic if (7.5) holds replacing genus 0 by genus $g$.

For $g$-sporadic, the list of (7.6) is too large. [GMS03, Thm. 4.1] tips off the adjustments for $g=1$ (\$7.2.4). For, $g>1, g$-sporadic groups should be just $A_{n} \leq G \leq S_{n}$ of $T_{n, 1}$ type, and cyclic or dihedral groups.

[GSh04] has 0-sporadics with an $A_{n}$ component. [FGMa02] has 0-sporadics groups with a rank 1 Chevalley group component. Magaard has written an outline of the large final step: Where components are higher rank Chevalley groups. Like the classification itself, someone going after a concise list of such examples for a particular problem will have difficulty culling the list for their problem. Various lists of the 0 -sporadics appear in many papers.

If someone outside group theory comes upon a problem suitable for the monodromy method or some other, can they go to these papers, look at the lists and finish their projects? [So01] has anecdotes and lists on the classification that many non-group theorists can read. Pointedly, however, is it sufficient to allow you or I to have replaced any contributor to [GMS03]? Unlikely! How about to read [GMS03]? Maybe! Yet, not without considerable motivation.

One needs familiarity with the relation between primitive subgroups of $S_{n}$ and simple groups, the description from Aschbacher-O'Nan-Scott. That does not rely on the classification. Rather, it treats simple group appearances as a black box. To decide if there are simple groups satisfying extra conditions contributing to the appearance of a particular primitive group, you must know special information about the groups in [So01, p. 341].

7.2.4. Qualitative versus quantitative. John's desire for documenting 0 -sporadic groups added many pages to the literature. What did particular examples do? How does one present specific examples to be useful? We have been suggesting $\S 2.4$ as a model. Mueller's list reveals just how relevant was Davenport's Problem for nailing polynomial 0-sporadic groups. Other examples, like [FGS93] and [GMS03], use a condition about a group normalizing $G$. This eliminated much of the primitive group classification. A seeker after applying the same method may find they, too, have such a useful condition, making it unnecessary to rustle through many of the lists like [FGMa02] or [GSh04]. We explain. Warning: An $\left(S_{5}, T_{5,2}\right)$ sporadic case occurs in answer a simple question about all indecomposable $f \in \mathbb{Z}[x]$ on Hilbert's irreducibility theorem. Nor can you just look at a 0 -sporadic Nielsen class to decide if it has an arithmetic property to be HIT-sporadic (a name coined for this occasion; [DFr99, $§ 2-\S 3])$. From experience, these sporadics, in service of a real problem, attract all the attention.

[GMS03, Thm. 1.4] classified 0-sporadics with the Schur property (Schursporadics) over number fields. In $[\mathbf{F r 8 0}$, p. 586$]$ we used the Schur property to show how to handle an entwining between arithmetic and geometric monodromy groups. Group theory setup: Two subgroups $G \leq \hat{G} \leq S_{n}$ of $S_{n}$ have this property.

(7.7) There is a $\tau \in \hat{G} \backslash G$ so that $g$ in the coset $G \tau$ implies $g$ fixes precisely one integer (see Ex.7.5).

[GMS03] calls our Schur covering property, arithmetic exceptionality.)

[GMS03, Thm. 1.4, c)] has the list where the genus of the Galois closure exceeds 1. These are the Schur-sporadics: Only finitely many 0-sporadic groups occur. Yet, John's original problem posed sporadic to mean the composition factors included other than cyclic or alternating groups. All three types of degree 25 alluded 
to above were not sporadic from this criterion. Indeed, the only nonsporadic from this criterion in the whole list were the groups $\mathrm{PSL}_{2}(n)$ with $n=8$ and 9 , and these had polynomial forerunners from [Mu95]. The more optimistic conjecture of $\$ 7.2 .3$ emerged because of the care John insisted upon.

Also, how about the nonsporadic appearances? Would you think big theorem when you hear of a study of dihedral groups? Yet, [Se68] is a big theorem. It is the arithmetic of special four branch point dihedral covers. The kind we call involution: They are in the Nielsen class $\mathrm{Ni}\left(D_{p^{k+1}}, \mathbf{C}_{2^{4}}\right)$, four repetitions of the involution conjugacy class in $D_{p^{k+1}}$. [Fr04, §5.2-5.3] connects this to exceptional covers (think Schur covering property). [Fr04, App. C] shows alternating and dihedral groups are dual for arithmetic questions about monodromy group covers.

EXAmPle 7.5 (Rational functions and the open image theorem). Notice that $D_{p} \leq \mathbb{Z} / p \times{ }^{s}(\mathbb{Z} / p)^{*} \leq S_{p}$ satisfies the criterion of (7.7). The goal is to describe rational functions $f \in K(x)$ with $K$ some number field so the Galois closure group $\hat{G}_{f}\left(\right.$ resp. $\left.G_{f}\right)$ of $f(x)-z$ over $K(z)$ (resp. $\mathbb{C}(z)$ ) gives such a pair. The only simplification is that $f$ can't decompose into lower degree polynomials over $K$. When $E$ is an elliptic curve without complex multiplication in $\S 6.2 .2$ produces $f$ indecomposable over $K$, but decomposable over $\mathbb{C}$. This is one of the two nonsporadic cases where the Galois closure cover for $f: \mathbb{P}_{w}^{1} \rightarrow \mathbb{P}_{z}^{1}$ has genus 1. [GMS03, Thm. 1.4, b)] has the complete list where the Galois closure cover has genus 1. A reader new to this will see some that look sporadic. Yet, those come from elliptic curves and topics like complex multiplication. They are cases where $K=\mathbb{Q}$ and there are special isogenies over $\mathbb{Q}$ defined by a $p$-division point not over $\mathbb{Q}$.

7.2.5. Monstrous Moonshine uniformizers. Recall a rough statement from Monstrous Moonshine. Most genus 0 quotients from modular subgroups of $\mathrm{PSL}_{2}(\mathbb{Z})$ have uniformizers from $\theta$-functions that are automorphic functions on the upper half plane. This inspired conjecture, to which John significantly contributed [To79a], gives away John's intense desire to see the genus 0 monodromy covers group theoretically. A recent Fields Medal to Borcherds on this topic corroborates the world's interest in genus 0 function fields, if the uniformizer has significance.

The Santa Cruz conference of 1979 alluded to on [So01, p. 341] ([Fe80] and [Fr80] came from there) had intense discussions of Monstrous Moonshine. This was soon after a suggestion by A. Ogg: He noticed that primes $p$ dividing the order of the Monster (simple group; denote it by $M$ ) are those where the function field of the normalizer of $\Gamma_{0}(p)$ in $\mathrm{PSL}_{2}(\mathbb{R})$ has genus zero. A. Pizer was present: He contributed that those primes satisfy a certain conjecture of Hecke relating modular forms of weight 2 to quaternion algebra $\theta$-series [P78]. Apparently Klein, Fricke and Hecke had recognized the problem of finding the function field generator of genus 0 quotients of the upper half-plane, not necessarily given by congruence subgroups of $\mathrm{PSL}_{2}(\mathbb{Z})$. It seems somewhere in the literature is the phrase genus 0 problem attached to a specific Hecke formulation.

Thompson concocted a relation between $q=e^{2 \pi i \tau}$-expansion coefficients of $j(\tau)=q^{-1}+\sum_{n=0}^{\infty} u_{n} q^{n}\left(u_{0}=744, u_{1}=196884, u_{2}=21493760, u_{3}=864299970\right.$, $\left.u_{4}=20245856256, u_{5}=333202640600\right)$ and irreducible characters of $M$. At the time, the Monster hadn't been proved to exist, and even if it did, some of its character degrees weren't shown for certain. ([To79a] showed if the Monster existed, these properties uniquely defined it.) John noted the coefficients listed for $j$ 
are sums of positive integral multiples of these. With this data he conjectured a $q$-expansion with coefficients in Monster characters with these properties [To79b]:

- the $q$ expansion of $j$ is its evaluation at 1 ; and

- the other genus 0 modular related covers have uniformizers from its evaluation at the other conjugacy classes of $M$.

[Ra00, p. 28] discusses those automorphic forms on the upper half plane with product expansions following Borcherd's characterization. Kac-Moody algebras give automorphic forms with a product expansion. The construction of a Monster Lie Algebra that gave $q$-expansions matching that predicted by Thompson is what - to a novice like myself — looks like the main story of [Ra00].

Is there any function, for example on any of the genus 0 spaces from this paper that vaguely has a chance to be like such functions? Between [BFr02, App. B.2] and [Ser90b] one may conclude the following discussion.

All components of the $\mathcal{H}_{p, k}^{\mathrm{rd}} \mathrm{s}$ in $\S 6.1$ have $\theta$-nulls canonically attached to their moduli definition. So do many of the quotients between $\mathcal{H}_{p, k+1}^{\mathrm{rd}}$ and $\mathcal{H}_{p, k}^{\mathrm{rd}}$. For the $\mathcal{H}_{p, k}^{\text {rd }}$ s we really do mean $\theta$-nulls defined by analytically continuing a $\theta$ function on the Galois cover $\varphi_{\boldsymbol{p}}: X_{\boldsymbol{p}} \rightarrow \mathbb{P}_{z}^{1}$ attached to $\boldsymbol{p} \in \mathcal{H}_{p, k}^{\mathrm{rd}}$, then evaluating it at the origin. Usually such $\theta$-nulls have a character attached to them. Here that would be related to the genus of $X_{\boldsymbol{p}}$. So, we cannot automatically assert these $\theta$-nulls are automorphic on the upper half plane (compare with genus 1 versions in [FaK01]). [Si63] (though hard to find) presents the story of $\theta$-functions defined by unimodular quadratic forms. These do define automorphic functions on the upper half plane.

The discussion for $\mathcal{H}_{2,1}$ alluded to two genus 1 and two genus 0 components. The $\theta$ for the genus 0 components is an odd function. So its $\theta$-null will be identically 0 . For the genus 1, components, however, it is even, and both those components cover (by a degree 2 map) a genus 0 curve between $\mathcal{H}_{2,1}$ and $\mathcal{H}_{2,0}$. That is one space we suggest for significance.

7.2.6. Strategies for success. [B03] has a portrait of Darwin as a man of considerable self-confidence, one who used many strategies to further his evolution theory. Though this is contrary to other biographies of Darwin, the case is convincing. Darwin was a voluminous correspondent, and his $14,000^{+}$letters are recorded in many places. Those letters reflected his high place in the scientific community. They often farmed out to his correspondent the task of completing a biological search, or even a productive experiment. So, great was Darwin's reputation that his correspondents allowed him to travel little (in later life), and yet accumulate great evidence for his mature volumes. Younger colleagues (the famous Thomas Huxley, for example) and even his own family (his son Francis, for instance) presented him a protective team and work companions. The success of the theory of evolution owes much to the great endeavor we call Charles Darwin.

[Ro03] reflects on the different way mathematical programs achieve success in his review of essays that touch on the growth of US mathematics into the international framework. He suggests the international framework that [PaR02] touts may not be the most compelling approach to analyzing mathematical success.

Recently, historians of science have tried to understand how ... locally gained knowledge produced by research schools becomes universal, a process that involves analyzing all the various mechanisms that produce consensus and support 
within broader scientific networks and communities. Similar studies of mathematical schools, however, have been lacking, a circumstance ... partly due to the prevalent belief that mathematical knowledge is from its very inception universal and ... stands in no urgent need to win converts.

There are two genus 0 problems: Problem $_{1}^{g=0}$ and Problem $_{2}^{g=0}$. They seem very different. Yet, they are two of the resonant contributions of John Thompson, outside his first area of renown. His influence on their solutions and applications is so large, you see I've struggled to complete their context. The historian remarks intrigue me for it would be valuable to learn, along their lines, more about our community.

\section{References}

[AFH03] W. Aitken, M. Fried and L. Holt, Davenport Pairs over finite fields, in proof, PJM, Dec. 2003.

[As90] M. Aschbacher, On conjectures of Guralnick and Thompson, J. Algebra 1990 \#2, $277-343$.

[AS85] M. Aschbacher and L. Scott, Maximal subgroups of finite groups, J. Alg. 92 (1985), $44-80$.

[A57] E. Artin, Geometric Algebra, Interscience tracts in pure and applied math. 3, 1957.

[BFr02] P. Bailey and M. Fried, Hurwitz monodromy, spin separation and higher levels of a Modular Tower, in Proceedings of Symposia in Pure Mathematics 70 (2002) editors M. Fried and Y. Ihara, 1999 von Neumann Conference on Arithmetic Fundamental Groups and Noncommutative Algebra, August 16-27, 1999 MSRI, 79-221.

[B03] J. Browne, Charles Darwin: The Power of Place, Knopf, 2003.

[Ca56] R.D. Carmichael, Introduction to the theory groups of finite order, Dover Pub. 1956.

[CoCa99] J.-M. Couveignes and P. Cassou-Noguès, Factorisations explicites de $g(y)-h(z)$, Acta Arith. 87 (1999), no. 4, 291-317.

[Co00] J.-M. Couveignes, Tools for the computation of families of covers, in Aspects of Galois Theory, Ed: H. Völklein, Camb. Univ. Press, LMS Lecture Notes 256 (1999), 38-65. The author informs me that due to a mistake of the editors, three pages have been cyclically permuted. The correct order of pages can be found following the numbering of formulae.

[CKS76] C.W. Curtis, W.M. Kantor and G.M. Seitz, The 2-transitive permutation representations of the finite Chevalley groups, TAMS 218 (1976), 1-59.

[DLS61] H. Davenport, D.J. Lewis and A. Schinzel, Equations of the form $f(x)=g(y)$, Quart. J. Math. Oxford 12 (1961), 304-312.

[DS64] H. Davenport, and A. Schinzel, Two problems concerning polynomials, Crelle's J. 214 (1964), 386-391.

[DDE04] P. Debes and M. Emsalem, Harbater-Mumford components and Towers of Moduli Spaces, Presentation by M. Emsalem at Graz, July 2003, preprint Jan. 2004.

[DFr94] P. Debes and M.D. Fried, Nonrigid situations in constructive Galois theory, Pacific Journal 163 \#1 (1994), 81-122.

[DFr99] P. Debes and M.D. Fried, Integral specializations of families of rational functions, PJM 190 (1999), 45-85.

[FaK01] H. Farkas and I. Kra, Theta Constants, Riemann Surfaces and the Modular Group, AMS graduate text series 37, 2001.

[Fe73] W. Feit, On symmetric balanced incomplete block designs with doubly transitive automorphism groups, J. of Comb.; Series A bf (1973), 221-247.

[Fe80] W. Feit, Some consequences of the classification of finite simple groups, Proceedings of Symposia in Pure Math: Santa Cruz Conference on Finite Groups, A.M.S. Publications 37 (1980), 175-181.

[Fe92] W. Feit, E-mail to Peter Müller, Jan. 28, 1992.

[Fr70] M.D. Fried, On a conjecture of Schur, Mich. Math. J. 17 (1970), 41-45.

[Fr73] M.D. Fried, The field of definition of function fields and a problem in the reducibility of polynomials in two variables, Ill. J. of Math. 17 (1973), 128-146. 
[Fr77] M. Fried, Fields of definition of function fields and Hurwitz families and groups as Galois groups, Communications in Algebra 5 (1977), 17-82.

[Fr78] M. Fried, Galois groups and Complex Multiplication, T.A.M.S. 235 (1978) 141-162.

[Fr80] M.D. Fried, Exposition on an Arithmetic-Group Theoretic Connection via Riemann's Existence Theorem, Proceedings of Symposia in Pure Math: Santa Cruz Conference on Finite Groups, A.M.S. Publications 37 (1980), 571-601.

[Fr95a] M. Fried, Extension of Constants, Rigidity, and the Chowla-Zassenhaus Conjecture, Finite Fields and their applications, Carlitz volume 1 (1995), 326-359.

[Fri95b] M. D. Fried, Modular towers: Generalizing the relation between dihedral groups and modular curves, Proceedings AMS-NSF Summer Conference, 186, 1995, Cont. Math series, Recent Developments in the Inverse Galois Problem, 111-171.

[Fr99] M.D. Fried, Separated variables polynomials and moduli spaces, Number Theory in Progress (Berlin-New York) (ed. J. Urbanowicz K. Gyory, H. Iwaniec, ed.), Walter de Gruyter, 1999, Proceedings of the Schinzel Festschrift, Summer 1997: Available from www.math.uci.edu/ mfried/\#math, 169-228.

[Fr02] M.D. Fried, Prelude: Arithmetic fundamental groups and noncommutative algebra, Proceedings of Symposia in Pure Mathematics, 70 (2002) editors M. Fried and Y. Ihara, 1999 von Neumann Conference on Arithmetic Fundamental Groups and Noncommutative Algebra, August 16-27, 1999 MSRI, vii-xxx.

[Fr04] M.D. Fried, Extension of constants series and towers of exceptional covers, preprint available in list at www.math.uci.edu/ ${ }^{\sim}$ mfried/\#math or www.math.uci.edu/ ${ }^{\sim}$ mfried/psfiles/exctow.html.

[Fr05] M.D. Fried, Riemann's existence theorem: An elementary approach to moduli, Chaps. 1-4 available at www.math.uci.edu/ ${ }^{\mathrm{mfried} / \# \text { ret. }}$

[FGS93] M.D. Fried, R. Guralnick and J. Saxl, Schur covers and Carlitz's conjecture, Israel J. 82 (1993), 157-225.

[FrS04] M.D. Fried and D. Semmen, Schur multiplier types and Shimura-like systems of varieties, preprint available in the list at www.math.uci.edu/ $\sim$ mfried/\#mt or www.math.uci.edu/ ${ }^{2}$ mfried/psfiles/schurtype.html.

[FV91] M. Fried and H. Völklein, The inverse Galois problem and rational points on moduli spaces, Math. Annalen 290 (1991), 771-800.

[FMa01] D. Frohardt and K. Magaard, Composition Factors of Monodromy Groups, Annals of Math. 154 (2001), 1-19

[FGMa02] D. Frohardt, R.M. Guralnick and K. Magaard, Genus 0 actions of groups of Lie rank 1, in Proceedings of Symposia in Pure Mathematics 70 (2002) editors M. Fried and Y. Ihara, 1999 von Neumann Conference on Arithmetic Fundamental Groups and Noncommutative Algebra, August 16-27, 1999 MSRI, 449-483.

[GHP88] L. Gerritzen, F. Herrlich, and M. van der Put, Stable n-pointed trees of projective lines, Ind. Math. 50 (1988), 131-163.

[GN92] R.M. Guralnick, The genus of a permutation group, in Groups, Combinatorics and Geometry, Ed: M. Liebeck and J. Saxl, LMS Lecture Note Series 165, CUP, Longdon, 1992.

[GMS03] R. Guralnick, P. Müller and J. Saxl, The rational function analoque of a question of Schur and exceptionality of permutations representations, Memoirs of the AMS 162 773 (2003), ISBN 0065-9266.

[GN95] R.M. Guralnick and M.G. Neubauer, Monodromy groups of branched coverings: the generic case, Proceedings AMS-NSF Summer Conference, 186, 1995, Cont. Math series, Ed: M. Fried Recent Developments in the Inverse Galois Problem, 325-352.

[GSh04] R.M. Guralnick and J. Shareshian, Symmetric and Alternating Groups as Monodromy Groups of Riemann Surfaces I, preprint.

[GT90] R.M. Guralnick and J.G. Thompson, Finite groups of genus 0, J. Algebra 131 (1990), 303-341.

[LS91] M. Liebeck and J. Saxl, Minimal degrees of primitive permutation groups, with an application to monodromy groups of covers of Riemann surfaces, PLMS (3) 63 (1991), 266-314.

[LSh99] M. Liebeck and A. Shalev, Simple groups, permutation groups, and probability, 497520 . 
[MM99] G. Malle and B.H. Matzat, Inverse Galois Theory, ISBN 3-540-62890-8, Monographs in Mathematics, Springer,1999.

[Mü98a] P. Müller, Kronecker conjugacy of polynomials, TAMS 350 (1998), 1823-1850.

[Mu95] P. Müller, Primitive monodromy groups of polynomials, Recent developments in the inverse Galois problem AMS, Cont. Math. Series Editor: Michael (1995), 385-401.

[PaR02] K. Parshall and A. Rice, Mathematics unbound: The evolution of an international mathematical research community, 1800-1945, History of Math. vol. 23, AMS/LMS, Prov. RI, 2002.

[P78] A. Pizer, A Note on a Conjecture of Hecke, PJM 79 (1978), 541-548.

[Ro03] D. Rowe, Review of [PaR02], BAMS 40 \#4 (2003), 535-542.

[Se68] J.-P. Serre, Abelian $\ell$-adic representations and elliptic curves, 1st ed., McGill University Lecture Notes, Benjamin, New York • Amsterdam, 1968, in collaboration with Willem Kuyk and John Labute.

[Ser90b] J.-P. Serre, Revêtements a ramification impaire et thêta-caractéristiques, C. R. Acad. Sci. Paris 311 (1990), 547-552.

[S91] T. Shih, A note on groups of genus zero, Comm. Alg. 19 (1991), 2813-2826.

[Si63] C.L. Siegel, Analytic Zahlentheorie II Vorlesungen, gehalten im Wintersemester 1963/64 an der Universität Göttingen, mimeographed notes.

[To79a] J.G. Thompson, Finite groups and modular functions, BLMS 11 (3) (1979), 347-351.

[To79b] J.G. Thompson, Some Numerology between the Fischer-Griess Monster and the elliptic modular function, BLMS 11 (3) (1979), 340-346.

[Ra00] U. Ray, Generalized Kac-Moody algebras and some related topics, BAMS 38 \#1, 1-42.

[So01] R. Solomon, A brief history of the classification of finite simple groups, BAMS $\mathbf{3 8}$ \# $\mathbf{3}$ (2001), 315-352.

[Vö96] H. Völklein, Groups as Galois Groups 53, Cambridge Studies in Advanced Mathematics, Camb. U. Press, Camb. England, 1996.

[We99] S. Wewers, Deformation of tame admissible covers of curves, in Aspects of Galois Theory, Ed: H. Völklein, Camb. Univ. Press, LMS Lecture Notes 256 (1999), 239-282.

[Wo64] K. Wohlfahrt, An extension of F. Klein's level concept, Ill. J. Math. 8 (1964), 529-535.

Emeritus, University of CALIFornia AT IRVINE

E-mail address: mfriedmath.uci.edu 